$12.18907 \&$ \&

DE91 004930

\title{
Deconvolution/Identification Techniques for 1-D Transient Signals
}

\author{
Dennis M. Goodman
}

October 1, 1990

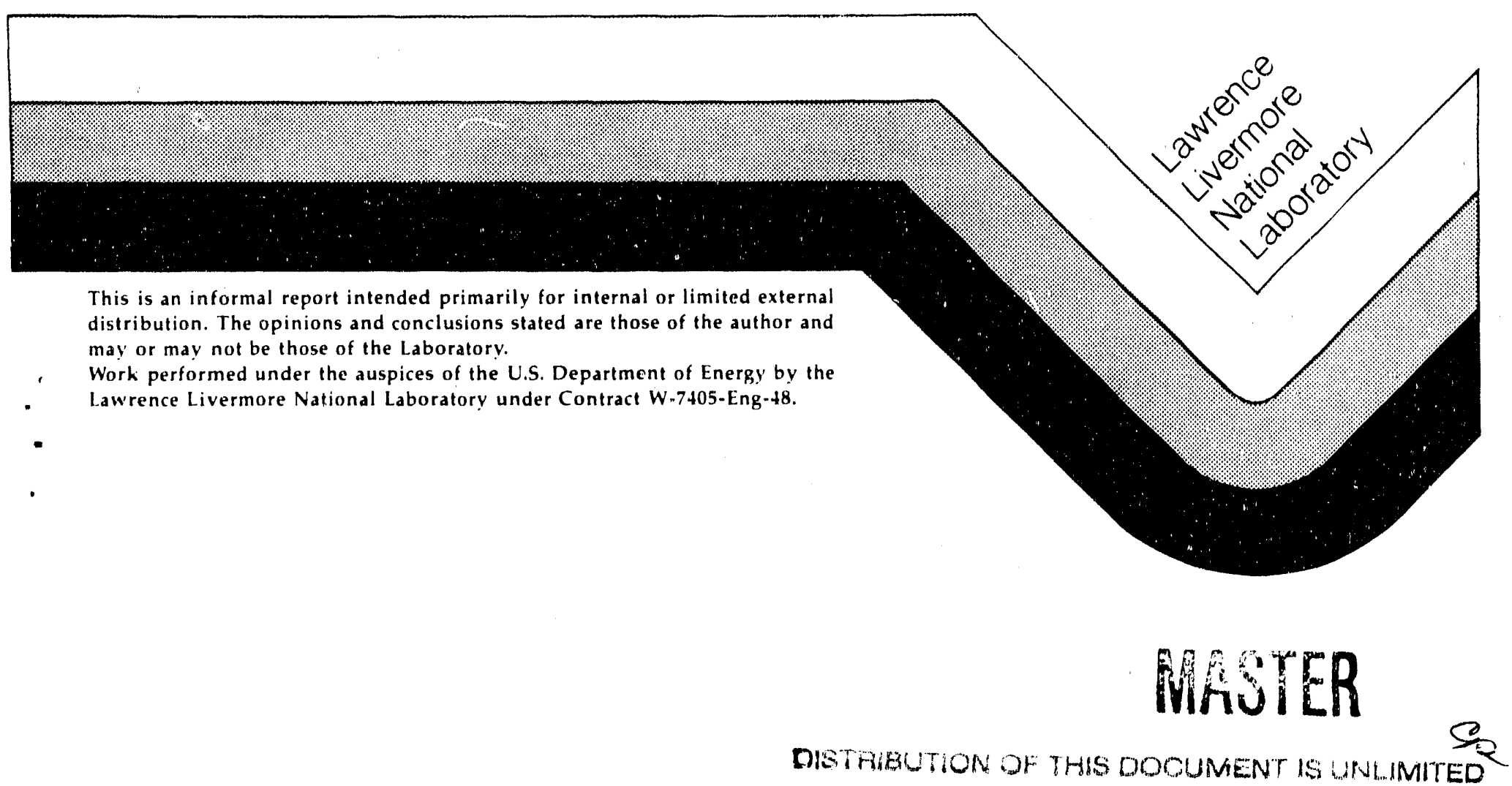


This document was prepared as an account of work sponsored by an agency of the United States Covernment. Neither the United States Government nor the University of Callfornia nor any of their employees, makes any warranty, express or implied, or assumes any legal liability or responsibility for the accuracy, completeness, or usefulness of any information, apparatus, product, or process disclosed, or represents that its use would not infringe privately owned rights. Reference herein to any specific commercial products, proress, or service by trade name, trademark, manufacturer, or otherwise, does not necessarily constitute or imply its endorsement, recommendation, or favoring by the United States Government or the University of Callfornia. The views and opinions of authors expressed herein do not necessarily state or reflect those of the United States Government or the University of Californiz, and shall not be used for advertising or product endorsement purposes.

This report has been reproduced
directly from the best available copy.

Available to DOE and DOE contractors from the

Office of Scientific and Technical Information

P.O. Box 02, Oak Ridge, TN 37831

Prices available from 16151 576-8401, FTS 626-8401.

Available to the public from the

National Technical Information Service

U.S. Department of Commerce

5285 Porl Royal Rd.

Springfield, VA 22161

Price

Code

A01

Papercopy Prices

A02

$\mathrm{A03}$

A04

A05

A06

A07

A08

A09

A10

A11

A12

A13

A14

A15

A16

A17

A18

A19

A20

A21

A22

A23

A24

A25

A99
Page

Range

Microfiche 


\title{
Deconvolution/Identiflcation Techniques for 1-D Transient Signals
}

\author{
Dennis M. Goodman \\ Laser Engineering Division
}

\begin{abstract}
This paper discusses a variety of nonparametric deconvolution and identification techniques that we have developed for application to 1-D transient signal problems. These methods are time-domain techniques that use direct methods for matrix inversion. Therefore, they are not appropriate for "large data" problems. These techniques involve various regularization methods and permit the use of certain kinds of a priori information in estimating the unknown. These techniques have been implemented in a package using standard FORT'RAN that should make the package readily transportable to most computers. This paper is also meant to be an instruction manual for the package.
\end{abstract}




\section{Introduction}

Two signal processing problems that occur frequently in a wide variety of scientific experiments are first: given noisy measurements of the response of a device to a known excitation, develop a general description of its input-output behavior so its response to an arbitrary excitation can be predicted, and second: given a description of a device's input-output behavior and noisy measurements of its response, determine the excitation. Often it is reasonable to assume that the device is linear so that its input-output behavior is completely determined by its impulse response. In this case the first problem is called impulse response identification, and the second deconvolution. Both problems come into play when removing the effects of a measurement system from experimental data. First, the system is characterized by applying a known input, measuring the output, and estimating its impulse response. Second, the identified impulse response is deconvolved from the experimental data. Typical measurement devices that introduce extra linear dynamics which must be removed before an experiment is analyzed include optical systems, acoustic transducers and electromagnetic probes. At first glance, solving these problems seems almost trivial-merely divide Fourier transforms. However, naive approaches to these problems often yield solutions that are very noisy, are highly biased, or both. We will discuss these issues in below, and we will try to make them more concrete by a detailed analysis of a deconvolution example in the final section.

The purpose of this paper is to discuss some nonparametric time-domain deconvolution/system identification algorithms that we have developed for 1-D transient signal problems. The same ideas developed here apply, theoretically at least, to steady state and higher dimensional cases as well, but the larger amounts of data in these cases would prohibit the use of the same numerical techniques we apply here. In particular, our algorithms are based on standard matrix inversion or factorization techniques that would be almost impossible to implement in large data cases. In such cases either iterative matrix methods (such as conjugate gradients) or frequency-domain methods are more appropriate. The 
model we use is

$$
\{y(n)\}=\{h(n)\} \star\{x(n)\}+\{e(n)\}
$$

where $\{h(n)\}$ is the kernel of the convolution operator, $\{x(n)\}$ is the input sequence, $\{e(n)\}$ is a noise sequence, and $\{y(n)\}$ is the observed, noise-corrupted output sequence. This model is shown in Fig. 1.

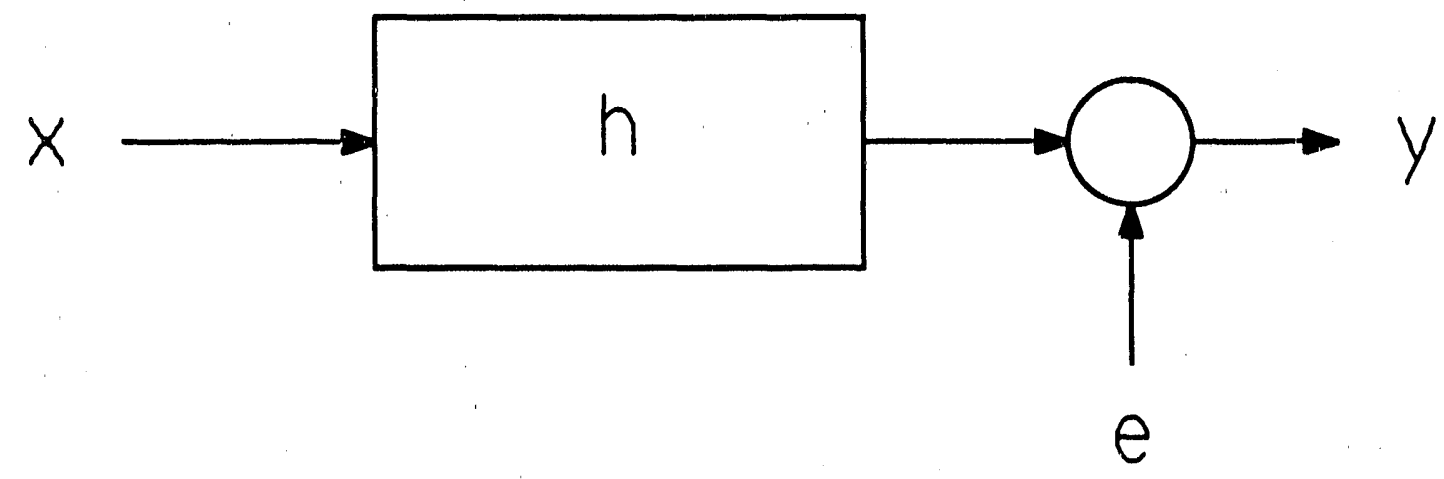

Fig. 1. Model of System

In the case of deconvolution, $\{h(n)\}$ is the known system impulse response and $\{x(n)\}$ is the input to be estimated, whereas in the case of nonparametric system identification, $\{x(n)\}$ is the known input and $\{h(n)\}$ is the system impulse response that is to be estimated. Because these problems are so similar, to save space we will consider only the deconvolution problem for the rest of this paper; our comments also apply to the identification problem. Note that the model assumes that the only noise appears at the observed output and that there is no noise connected with the convolution kernel $\{h(n)\}$; it is assumed to be known exactly. The assumption of output noise only is usually reasonable. However, if $\{h(n)\}$ is noisy, the problem of estimating $\{x(n)\}$ becomes considerably more difficult. Noise associated with $\{h(n)\}$ that is not modeled when estimating $\{x(n)\}$ can introduce a bias (i.e., a consistent error) in the estimate of $\{x(n)\}$. Therefore, the algorithms we discuss here may not be appropriate if there is considerable uncertainty associated with $\{h(n)\}$. A discussion of bias problems for this "errors in the variables case" is given in 
Section 7-14 of [1]. Just how difficult this problem could be is suggested by the discussion in Ref. [2] where total least squares is presented as a method for dealing with errors in $\{h(n)\}$. In particular, the total least squares estimate has the form of the estimate shown in Eq. (13) below except that removing the bias calls for a negative $\lambda$. Consequently, total least squares must be viewed as a deregularization technique that is at odds with everything we present here.

Although the mociel of Eq. (1) is usually appropriate, even in this simplified case obtaining a good estimate of $\{x(n)\}$ is often difficult. The two most serious problems are ill-posedness and data truncation. The problem of inverting Eq. (1) is usually by its very nature ill-posed because it is a discretization of a Fredholm integral equation of the first kind [3]. The methods we present deal with ill-posedness by constraining the solution. Often it is necessary to truncate data records obtained from a transient experiment either because after some time the data becomes corrupted by reflections or other spurious signals, or because the collection time of the recording device is limited. Frequency-domain methods assume that data outside the known records are zero, and the result is often a highly biased estimate. The advantage of time-domain methods is that the part of the convolution equation that extends beyond the known data can be eliminated from the problem. If the length of the kernel is $N$, and the length of the unknown is assumed to be $M$, then the length of the output is $N+M-1$. Consider the special case in which the kernel and the unknown both have length $\mathrm{N}$ and are zero elsewhere: the convolution equation can be written in matrix form as

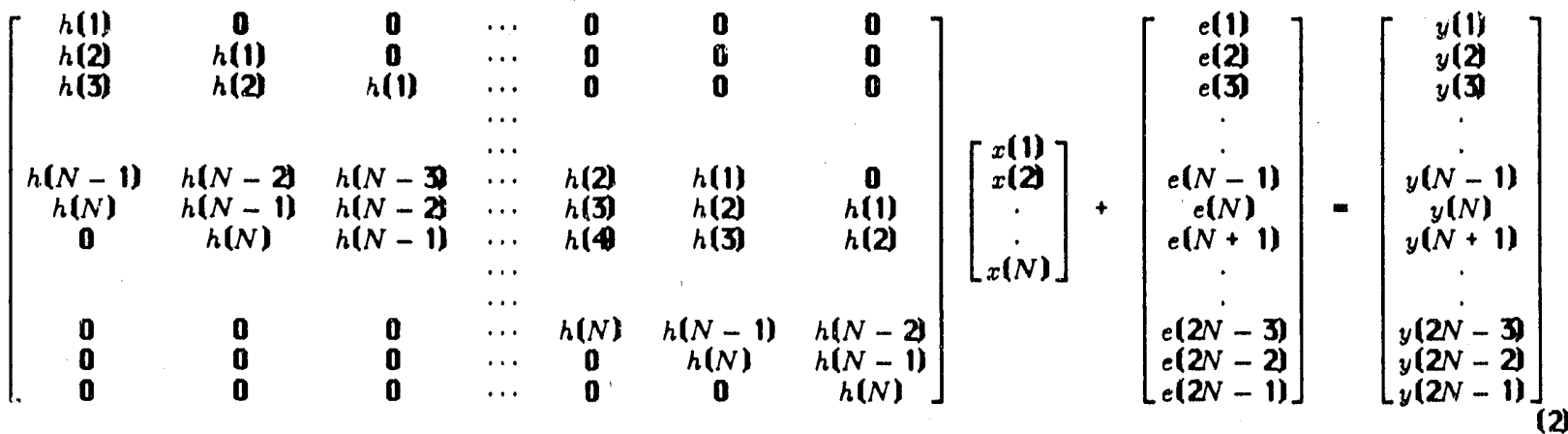


In a typical experiment, both the kernel and the output are collected by observing the system under test, both have the same length $N$, and the length of the unknown is also $N$. This situation causes a conflict with Eq. (2) because $y(N+1), \ldots, y(2 N-1)$ are not available. With frequency-domain methods it is necessary to assume that these points are zero, and the result is of ten a highly biased estimate if the signals have not died out by the $N^{\text {th }}$ sample. With time-domain methods this problem can be avoided by eliminating the last $N-1$ rows of Eq. (2). This removes $y(N+1), \ldots, y(2 N-1)$ from the equations, thereby eliminating any assumption whatever about their values. Assuming that the output data beyond the $N^{\text {th }}$ sample is zero is called "postwindowing." It is implicitly assumed in Eq. (2) that the output is zero before the first sample. Here again, this assumption is necessary in frequency-domain methods, but can be avoided by using a time-domain method. In particular, removing the first $N-1$ rows of Eq. (2) eliminates any assumptions about the output data before the first sample. Assuming that the output data is zero before the first sample is called "prewindowing." For most transient experiments the system is assumed to be in a quiescent state before the experiment starts, and prewindowing is reasonable. On the other hand, if the data records are truncated before the signals have died out, postwindowing is usually unreasonable.

The code asks for the length of the kernel record, the length of the output record, and the length of the unknown. In addition the user is given the options to or not to prewindow and postwindow. All of these options can get the user in trouble if he does not think carefully about what he is doing. In the example above where all of the lengths are $N$, not prewindowing removes all but the last sample of the output record from the equations; if in addition the equations are not postwindowed, there will only be one equation in $N$ unknowns! On the other hand, prewindowing but not postwindowing includes all $N$ known output samples in the equations, and gives $N$ equations in $N$ unknowns. For "sanity checks," the code tells the user the number of equations in the number of unknowns and which output samples are included in the equations. Probably the most important issue 
that the user should keep in mind is that not prewindowing eliminates the first part of the output record from the equations. For many transient experiments, it is this part that is most significant.

\section{Reducing Error Variance Via Regularization}

An inverse problem is said to be ill-posed if small changes in the observed data can produce large changes in a quantity estimated from the data. For our problems ill-posedness is usually a consequence of the fact that the known kernel has zeros or near zeros in its frequency response. Regularization improves the behavior of estimates by restricting the class of possible solutions. The method we employ is to require that any solution have a weighted norm that is less than some constant.

The convolution equation can be written in matrix form as

$$
\mathbf{H x}+\mathbf{e}=\mathbf{y}
$$

where, as in Eq. (2), $\mathrm{H}$ is a Hankel matrix whose columns are shifted versions of the kernel, $\mathbf{x}$ is a vector formed by the unknown sequence, $\mathbf{e}$ is the noise sequence vector, and $\mathbf{y}$ is the observed output sequence vector. The dimensions of the matrix and the vectors in this equation depend, of course, on the lengths of the various sequences and the decisions about windowing. Here we assume that $\mathbf{H}$ is $n \times p$ so that $\mathbf{x}$ is a $p$ vector, and $\mathbf{e}$ and $\mathbf{y}$ are $n$ vectors.

To make the analysis easier, we make the usual assumption that the noise sequence is zero mean, Gaussian and white. In particular, the covariance matrix of $e$ is

$$
E\left\{\mathrm{ee}^{T}\right\}=\sigma^{2} \mathbf{I}
$$

where $I$ is the identity matrix. This is usually a reasonable assumption. If the noise is not white, prewhitening or weighted least squares can be used in what follows, but the presentation is more complicated, so we assume whiteness. Under these assumptions, the minimum variance unbiased estimator of $\mathbf{x}$ is the vector $\hat{\mathbf{x}}$ that minimizes the fit of $\mathbf{H} \hat{\mathbf{x}}$ to 
$y$ in the least-squares sense, i.e.,

$$
\hat{\mathbf{x}}=\underset{\mathbf{A}}{\operatorname{Argmin}}\left\{[\mathbf{y}-\mathbf{H} \hat{\mathbf{x}}]^{T}[\mathbf{y}-\mathbf{H} \hat{\mathbf{x}}]\right\} .
$$

Assuming that $\mathbf{H}$ has full rank, the solution to minimizing Eq. (5) is

$$
\hat{\mathbf{x}}=\left(\mathbf{H}^{T} \mathbf{H}\right)^{-1} \mathbf{H}^{T} \mathbf{y} .
$$

Unfortunately, due to the ill-posed nature of the deconvolution/identification problem, this estimator usually behaves poorly. In particular, it is easy to show [4] that the covariance matrix of $\hat{\mathbf{x}}$ is

$$
E[\hat{\mathbf{x}}-\mathbf{x}][\hat{\mathbf{x}}-\mathbf{x}]^{T}=\sigma^{2}\left(\mathbf{H}^{T} \mathbf{H}\right)^{-1}
$$

and, as claimed, this estimate is unbiased: $E\{\hat{\mathbf{x}}\}=\mathbf{x}$. Consequently, the expected total mean-square error in this estimate is

$$
E[\hat{\mathbf{x}}-\mathbf{x}]^{T}[\hat{\mathbf{x}}-\mathbf{x}]=\sigma^{2} \operatorname{Trace}\left\{\left(\mathbf{H}^{T} \mathbf{H}\right)^{-1}\right\}=\sigma^{2} \sum_{m=1}^{p} \frac{1}{\lambda_{m}},
$$

where $\lambda_{1}, \ldots, \lambda_{p}$ are the eigenvalues of $\mathbf{H}^{T} \mathbf{H}$. The size of these eigenvalues is closely related to the energy density spectrum of the kernel sequence. As was noted in [5], if the energy density spectrum was nearly zero over some range of frequencies, then the problem is ill-posed, there will be eigenralues that are close to zero, and the mean-square error will be very high.

An idea of what is wrong and what to do about it can be obtained by rewriting Eq. (8) as

$$
E\left\{\hat{\mathbf{x}}^{T} \hat{\mathbf{x}}\right\}=\mathbf{x}^{T} \mathbf{x}+\sigma^{2} \sum_{m=1}^{p} \frac{1}{\lambda_{m}} .
$$

This equation illustrates the seemingly paradoxical fact that the best unbiased estimator can be expected to give an estimate whose energy is much larger than that of the true $\mathbf{x}$. This suggests that to obtain a better estimate a constraint on the size of $\hat{\mathbf{x}}$ should be included in the estimation problem; instead of solving Eq. (5), we solve:

$$
\begin{aligned}
\hat{\mathbf{x}}= & \underset{\mathbf{a}}{\operatorname{Argmin}}\left\{[\mathbf{y}-\mathbf{H} \hat{\mathbf{x}}]^{T}[\mathbf{y}-\mathbf{H} \hat{\mathbf{x}}]\right\} \\
& \text { subject to: }[\mathbf{F} \hat{\mathbf{x}}]^{T}[\mathbf{F} \hat{\mathbf{x}}] \leq k,
\end{aligned}
$$


where $k$ is some constant that must be determined. Restricting the set of solutions in this way is the form of regularization considered in this paper. The selection of an appropriate F is an important issue. The code uses matrices that can be defined by convolutional relationships: $\mathbf{F}$ has the same Hankel structure as $\mathbf{H}$ in that its columns are shifted versions of a filter sequence $\{f(n)\}$. Observe that because $\mathbf{F}$ involves constraining the solution, to smooth the solution a high-pass filter kernel should be used. Currently the user has the option of zeroth-order smoothing for which $\{f(n)\}=\{1\}$, first-order smoothing for which $\{f(n)\}=\{-1,1\}$, and second-order smoothing for which $\{f(n)\}=\{1,-2,1\}$. We are currently installing the filter package from [6] to provide the user with more flexibility. The prewindowing and postwindowing options are also provided for the filter kernel. For the typical transient experiment, one should always prewindow and use careful judgement on postwindowing. In particular, assuming that the unknown is zero outside the estimation interval will cause the first or second order smoother to try to force the estimate to zero at the ends of the known interval. Therefore, if the true value of the unknown is not zero at the ends, the result could be a large amount of bias in the estimate.

Using Lagrange multipliers shows that the solution to Eq. (10) is also the solution to

$$
\hat{\mathbf{x}}=\underset{\mathbf{R}}{\operatorname{Argmin}}\left\{[\mathbf{y}-\mathbf{H} \hat{\mathbf{x}}]^{T}[\mathbf{y}-\mathbf{H} \hat{\mathbf{x}}]+\lambda[\mathbf{F} \hat{\mathbf{x}}]^{T}[\mathbf{F} \hat{\mathbf{x}}]\right\}
$$

and to the augmented least-squares problem

$$
\left[\begin{array}{r}
\mathbf{H} \\
\sqrt{\lambda} \mathbf{F}
\end{array}\right] \hat{\mathbf{x}} \stackrel{\text { l.s. }}{\approx}\left[\begin{array}{l}
\mathbf{y} \\
\mathbf{0}
\end{array}\right]
$$

In Eqs. (11) and (12) $\lambda$ is a Lagrange multiplier that is a monotonically decreasing function of $k$. The solution to these equations is

$$
\hat{\mathbf{x}}(\lambda)=\left(\mathbf{H}^{T} \mathbf{H}+\lambda \mathbf{F}^{T} \mathbf{F}\right)^{-1} \mathbf{H}^{T} \mathbf{y}
$$

Note that we have expressed the dependence of the estimate on $\lambda$, rather than $k$; this is because most methods that determine the optimal degree of smoothing estimate a value for $\lambda$. Indeed, we will henceforth call $\lambda$ the regularization parameter. 
The reader at this point may be asking what is the relationship between regularization and Bayesian estimation in which a prior probability density function is assigned to the unknown. We wish to emphasize that we are not directly using Bayesian estimation. However, it may be informative to observe that if a certain prior is assigned to the unknown, then the form of the resulting estimate will be the same as ours. In particular, suppose that we assign the prior density

$$
N\left(\mathbf{0}, \lambda^{-1} \sigma^{2}\left[\mathbf{F}^{T} \mathbf{F}\right]^{-1}\right)
$$

to $\mathbf{x}$; i.e., $\mathbf{x}$ is zero mean multivariate Gaussian with covariance matrix $\lambda^{-1} \sigma^{2}\left[\mathbf{F}^{T} \mathbf{F}\right]^{-1}$, where $\sigma^{2}$ is the variance of the output noise $\{e(n)\}$. Then the Bayes estimate of $\mathbf{x}$ is given by Eq. (13). Of course, for the regularization problem $\lambda$ is not available and must be determined from the data. Within a Bayesian framework this approach is called empirical Bayes analysis [7]. Methods for determining a good value for $\lambda$ are covered in the next section.

In many deconvolution/identification problems the unknown is known to be nonnegative. The most obvious example is the case where the unknown is energy or power, as in incoherent imaging systems. Further regularizing the problem by restricting the class of solutions to be nonnegative frequently leads to greatly improved estimates. We modify Eq. (10) by seeking the solution to

$$
\begin{aligned}
\hat{\mathbf{x}}= & \underset{\mathbf{A}}{\operatorname{Argmin}}\left\{[\mathbf{y}-\mathbf{H} \hat{\mathbf{x}}]^{T}[\mathbf{y}-\mathbf{H} \hat{\mathbf{x}}]\right\} \\
& \text { subject to: }\left\{\begin{array}{c}
{[\mathbf{F} \hat{\mathbf{x}}]^{T}[\mathbf{F} \hat{\mathbf{x}}] \leq k} \\
\hat{\mathbf{x}} \geq \mathbf{0}
\end{array}\right.
\end{aligned}
$$

Using the Kuhn-Tucker conditions [8], we see that the solution to Eq. (15) is also the solution to the constrained, augmented least-squares problem

$$
\begin{aligned}
& {\left[\begin{array}{r}
\mathbf{H} \\
\sqrt{\lambda} \mathbf{F}
\end{array}\right] \hat{\mathbf{x}} \stackrel{\text { l.s. }}{\approx}\left[\begin{array}{l}
\mathbf{y} \\
\mathbf{0}
\end{array}\right]} \\
& \text { subject to: } \hat{\mathbf{x}} \geq 0,
\end{aligned}
$$

where $\lambda$ is the usual regularization parameter. 


\section{Choosing a Good Value for the Regularization Parameter}

In the previous sections we developed the form of our estimators. All depend critically on the choice of a good value for the regularization parameter $\lambda$. There is a large variety of possible methods. We provide a selection of what we believe are the most reasonable in this section. All of the methods we describe are provided for in the code. We first discuss how our methods apply when $\hat{\mathbf{x}}$ does not have a sign constraint, and then we discuss how they extend to the case where $\hat{\mathbf{x}} \geq 0$.

The first method is the discrepancy principle in which $\lambda$ is adjusted to make the total energy in the residuals match the enorgy that one would expect the residuals to have given the amount of noise present on the output [3]. Given that there are $n$ equations, the total expected residual energy due to noise is $n \sigma^{2}$; hence, $\lambda$ is adjusted to satisfy:

$$
[\mathbf{y}-\mathbf{H} \hat{\mathbf{x}}(\lambda)]^{T}[\mathbf{y}-\mathbf{H} \hat{\mathbf{x}}(\lambda)]=n \sigma^{2}
$$

where $\hat{\mathbf{x}}(\lambda)$ is the solution to either Eq. (12), or, if nonnegativity has been imposed, Eq. (16). Although the arguments for this method are mostly intuitive, there are at least some good theoretical reasons for its use. The most important is that under certain conditions the method is consistent: as $n$ gets large the discrepancy principle estimate approaches the true $\mathbf{x}$. For finite amounts of data, the method tends to oversmooth; i.e., the estimated value of $\lambda$ is too large.

Unfortunately, the value of the output noise variance, $\sigma^{2}$, may be unknown to the experimenter. Instead, he may have an intuitive idea of the number of significant digits or the percentage error in the observed output. To allow for this, the code has an option that permits the user to type in the percentage error, $\alpha$, and adjusts $\lambda$ to satisfy

$$
[\mathbf{y}-\mathbf{H} \hat{\mathbf{x}}(\lambda)]^{T}[\mathbf{y}-\mathbf{H} \hat{\mathbf{x}}(\lambda)]=(.01 a)^{2} \mathbf{y}^{T} \mathbf{y}
$$

A second method adjusts $\lambda$ to provide an estimate whose sum-of-squares, $\gamma$, is a specified value. Although there may be some estimation problems for which the energy in the 
unknown can be specified, a more common use for this method is the signal synthesis problem. For example, suppose that given a known impulse response and a desired output it is necessary to determine an input that will yield an approximation to the desired output. The energy constraint is imposed to meet physical limitations on the equipment. The code selects $\lambda$ to satisfy

$$
\hat{\mathbf{x}}(\lambda)^{T} \hat{\mathbf{x}}(\lambda)=\gamma
$$

Methods three and four are prediction error methods that attempt to mininize the function $\mathbf{H x}-\mathbf{H} \hat{\mathbf{x}}(\lambda)$ in a mean-square sense; in other words, we select $\lambda$ to make $\mathbf{H} \hat{\mathbf{x}}(\lambda)$ a good estimate of the nuise-free output. Because such methods attempt to minimize the error in the range of the model, rather than in its domain, the term frequently used in statistical literature to describe prediction error methods is range risk. Of course, whit we would really like is the $\lambda$ that minimizes $\mathbf{x}-\hat{\mathbf{x}}(\lambda)$. However $\mathbf{x}$ is the unknown we are estimating, so minimizing this function directly would be absurd because we would be estimating what we already know. Surprisirgly, there are estimates of the prediction error that do not require knowledge of the unknown; methods three and four pick $\lambda$ to minimize one of these estimates. By writing the prediction error as $\mathbf{H}[\mathbf{x}-\hat{\mathbf{x}}(\lambda)]$ we see that prediction error is a weighted measure of $\mathbf{x}-\hat{\mathbf{x}}(\lambda)$. The theory for the ronnegative constraint case is not complete, so we derive the unconstrained case first; then we extend the ideas to the constrained case. To explain how such methods work, it is probably best to first backtrack a bit and explain some of the philosophy behind regularization and show why it could be expected to work. The total mean-square error in an estimate $\hat{\mathbf{x}}$ of $\mathbf{x}$ is $E\left\{[\hat{\mathbf{x}}-\mathbf{x}]^{T}[\hat{\mathbf{x}}-\mathbf{x}]\right\}$. If the expected value of the estimate is $E\{\hat{\mathbf{x}}\}=\overline{\mathbf{x}}$, then

$$
\begin{aligned}
E\left\{[\hat{\mathbf{x}}-\mathbf{x}]^{T}[\hat{\mathbf{x}}-\mathbf{x}]\right\} & =E\left\{[\hat{\mathbf{x}}-\overline{\mathbf{x}}]^{T}[\hat{\mathbf{x}}-\overline{\mathbf{x}}]\right\}+[\mathbf{x}-\overline{\mathbf{x}}]^{T}[\mathbf{x}-\overline{\mathbf{x}}] \\
& =\text { estimator variance }+(\text { estimator bias) }
\end{aligned}
$$

From this equation we see inat mean-square error can be separated into two parts: the variance and the bias. The estimator variance is a measure of how much an estimate will 
change from data set to data set, and it can be reduced by averaging estimates froru many data sets. On the other hand, a bias is a consistent error that will not be reduced by averaging.

Consider the estimate given by Eq. (13). If we define

$$
\mathbf{L}(\lambda)=\left(\mathbf{H}^{T} \mathbf{H}+\lambda \mathbf{F}^{T} \mathbf{F}\right)^{-1} \mathbf{H}^{T},
$$

Then using Eq. (3) to substitute for the observed output gives

$$
\hat{\mathbf{x}}(\lambda)=\mathbf{L}(\lambda)(\mathbf{H} \mathbf{x}+\mathbf{e})
$$

Recalling our assumptions about output noise and taking expectation gives

$$
\overline{\mathbf{x}}(\lambda)=E\{\hat{\mathbf{x}}(\lambda)\}=\mathbf{L}(\lambda) \mathbf{H} \mathbf{x} .
$$

This can then be used to give the following expression's for the mean-square error in terms of the variance:

$$
\begin{aligned}
E\left\{[\hat{\mathbf{x}}(\lambda)-\overline{\mathbf{x}}(\lambda)]^{T}[\hat{\mathbf{x}}(\lambda)-\overline{\mathbf{x}}(\lambda)]\right. & =\operatorname{Trace}\left\{E\{[\hat{\mathbf{x}}(\lambda)-\overline{\mathbf{x}}(\lambda)][\hat{\mathbf{x}}(\lambda)-\overline{\mathbf{x}}(\lambda)]\}^{T}\right. \\
& =\operatorname{Trace}\left\{E\left\{\mathbf{L}(\lambda) \mathbf{e e}^{T} \mathbf{L}(\lambda)^{T}\right\}\right\} \\
& =\sigma^{2} \operatorname{Trace}\left\{\mathbf{L}(\lambda) \mathbf{L}(\lambda)^{T}\right\}
\end{aligned}
$$

and the bias squared:

$$
[\mathbf{x}-\overline{\mathbf{x}}]^{T}[\mathbf{x}-\overline{\mathbf{x}}]=\mathbf{x}^{T}[\mathbf{I}-\mathbf{L}(\lambda) \mathbf{H}]^{T}[\mathbf{I}-\mathbf{L}(\lambda) \mathbf{H}] \mathbf{x}
$$

For $\lambda=0$, the bias term is zero and we have the best unbiased estimator discussed previously. If $\mathbf{F}$ is full rank, then as $\lambda$ becomes large the variarice decreases to zero and the bias squared increases to the upper bound of $\mathbf{x}^{T} \mathbf{x}$. Therefore, the problem of selecting $\lambda$ is a bias vs. variance tradeoff. It is possible to show [9] that there is always a $\lambda \geq 0$ that gives a lower mean-squared error than the best unbiased estimator. In othes words, there is always a biased estimator with less mean-squared error than the best unbiased 
estimator. However, as we noted earlier, it is not possible to minimize Eq. (20) directly. In particular, note that although the unknown $\mathbf{x}$ does not appear in the variance terri, it does appear in the bias-squared term.

From Eqs. (24) and (25) we see that the mean-square prediction error can be written in terms of the variance and the bias squared as

$$
\begin{aligned}
E\left\{[\mathbf{H}(\mathbf{x}-\hat{\mathbf{x}}(\lambda))]^{T}[\mathbf{H}(\mathbf{x}-\hat{\mathbf{x}}(\lambda))]\right\}= & E\left\{[\mathbf{H}(\hat{\mathbf{x}}-\overline{\mathbf{x}})]^{T}[\mathbf{H}(\hat{\mathbf{x}}-\overline{\mathbf{x}})]\right\} \\
& +[\mathbf{H}(\mathbf{x}-\overline{\mathbf{x}})]^{T}[\mathbf{H}(\mathbf{x}-\overline{\mathbf{x}})] \\
= & \sigma^{2} \operatorname{Trace}\left\{\mathbf{H L}(\lambda) \mathbf{L}(\lambda)^{T} \mathbf{H}^{T}\right\} \\
& +\mathbf{x}^{T}[\mathbf{I}-\mathbf{I}(\lambda) \mathbf{H}]^{T} \mathbf{H}^{T} \mathbf{H}[\mathbf{I}-\mathbf{L}(\lambda) \mathbf{H}] \mathbf{x}(26)
\end{aligned}
$$

Because the unknown appears in the bias term of this equation also, it can't be minimized directly either. Fortunately, however, there is an unbiased estimate of mean-square prediction error that can be calculated from data. This estimator depends on the residual sum of squares, which we denote by $\operatorname{RSS}(\lambda)$ The residual vector is the mismatch between the observed output, $\mathbf{y}$, and the "model" $\mathbf{H} \hat{\mathbf{x}}(\lambda)$ :

$$
\begin{aligned}
\mathbf{y}-\mathbf{H} \hat{\mathbf{x}}(\lambda) & =[\mathbf{I}-\mathbf{H L}(\lambda)] \mathbf{y} \\
& =[\mathbf{I}-\mathbf{H L}(\lambda)][\mathbf{H} \mathbf{x}+\mathbf{e}] .
\end{aligned}
$$

Consequently,

$$
\begin{aligned}
\operatorname{RSS}(\lambda) & =[\mathbf{y}-\mathbf{H} \hat{\mathbf{x}}(\lambda)]^{T}[\mathbf{y}-\mathbf{H} \hat{\mathbf{x}}(\lambda)] \\
& =[\mathbf{H} \mathbf{x}+\mathbf{e}]^{T}[\mathbf{I}-\mathbf{H L}(\lambda)]^{T}[\mathbf{I}-\mathbf{H L}(\lambda)][\mathbf{H} \mathbf{x}+\mathbf{e}]
\end{aligned}
$$

Taking expectation gives:

$$
\begin{aligned}
E\{\operatorname{RSS}(\lambda)\}= & \mathbf{x}^{T} \mathbf{H}^{T}[\mathbf{I}-\mathbf{H L}(\lambda)]^{T}[\mathbf{I}-\mathbf{H L}(\lambda)] \mathbf{H} \mathbf{x}+E\left\{\mathbf{e}^{T}[\mathbf{I}-\mathbf{H L}(\lambda)]^{T}[\mathbf{I}-\mathbf{H L}(\lambda)] \mathbf{e}\right\} \\
= & \mathbf{x}^{T}[\mathbf{I}-\mathbf{L}(\lambda) \mathbf{H}]^{T} \mathbf{H}^{T} \mathbf{H}[\mathbf{I}-\mathbf{L}(\lambda) \mathbf{H}] \mathbf{x}+\operatorname{Trace}\left\{E\left\{[\mathbf{I}-\mathbf{H L}(\lambda)] \mathbf{e} \mathbf{e}^{T}[\mathbf{I}-\mathbf{H L}(\lambda)]^{T}\right\}\right\} \\
= & \mathbf{x}^{T}[\mathbf{I}-\mathbf{L}(\lambda) \mathbf{H}]^{T} \mathbf{H}^{T} \mathbf{H}[\mathbf{I}-\mathbf{L}(\lambda) \mathbf{H}] \mathbf{x} \\
& +n \sigma^{2}+\sigma^{2} \operatorname{Trace}\left\{\mathbf{H L}(\lambda) \mathbf{L}(\lambda)^{T} \mathbf{H}^{T}\right\}-2 \sigma^{2} \operatorname{Trace}\{\mathbf{H L}(\lambda)\}
\end{aligned}
$$


Using Eq. (29). to substitute for the bias squared term in Eq. (26) gives

$$
E\left\{[\mathbf{H}(\mathbf{x}-\hat{\mathbf{x}}(\lambda))]^{T}[\mathbf{H}(\mathbf{x}-\hat{\mathbf{x}}(\lambda))]\right\}=E\{\operatorname{RSS}(\lambda)\}-n \sigma^{2}+2 \sigma^{2} \operatorname{Trace}\{\mathbf{H L}(\lambda)\}
$$

It follows that the statistic

$$
\operatorname{RSS}(\lambda)-n \sigma^{2}+2 \sigma^{2} \operatorname{Trace}\{\mathbf{H L}(\lambda)\}
$$

is an unbiased estimator of the prediction error; furthermore, it can be calculated from the data. In [10] we discussed minimizing a normalized version of this statistic as a way to determine $\lambda$ for identification problems. This is our third method for determining $\lambda$. This method for determining $\lambda$ for the general linear regression problem was first derived in [11], where Eq. (31) normalized by $\sigma^{2}$ was called the $C_{L}$ statistic:

$$
C_{L}(\lambda)=\frac{\operatorname{RSS}(\lambda)}{\sigma^{2}}+\frac{2}{\sigma^{2}} \operatorname{Trace}\{\mathbf{H L}(\lambda)\}-n .
$$

The reason for using a normalized version is that the method proposed by Mallows is a graphical technique that involves plotting $C_{L}(\lambda)$ vs. another statistic that also depends on $\lambda$; we do not discuss this method here. Clearly, the minima of Eqs. (31) and (32) are the same. Statistical properties of Eq. (31) that argue for its use are derived in [12].

Our fourth method for selecting $\lambda$ is Generalized Cross Validation (GCV). GCV is a prediction error method that does not require a-priori knowledge of $\sigma^{2}$. More detailed discussions of GCV than we will give below are contained in [13] and [14]. GCV is closely related to the third method derived above. In order to be consistent with the notation used in [13] and [14] we normalize Eq. (31) by $n$, rather than $\sigma^{2}$, to define

$$
\hat{T}(\lambda)=\frac{1}{n} \operatorname{RSS}(\lambda)+\frac{2}{n} \sigma^{2} \operatorname{Trace}\{\mathbf{H L}(\lambda)\}-\sigma^{2} .
$$

Define $\mathbf{h}_{\boldsymbol{i}}$ to be the $i^{\text {th }}$ row of $\mathbf{H}$ and $\mathbf{H}_{\boldsymbol{i}}$ to be the $\mathbf{H}$ matrix with its $i^{\text {th }}$ row deleted. Furthermore, define $\mathbf{y}_{i}$ to be $\mathbf{y}$ with its $i^{\text {th }}$ elemert, $y_{i}$, deleted. Let $\hat{\mathbf{x}}_{i}(\lambda)$ be the estimate of $\mathbf{x}$ computed with the $i^{\text {th }}$ equation of the convolution relationship deleted; i.e.,

$$
\hat{\mathbf{x}}_{i}(\lambda)=\left(\mathbf{H}_{i}^{T} \mathbf{H}_{i}+\lambda \mathbf{F}^{T} \mathbf{F}\right)^{-1} \mathbf{H}_{i}^{T} \mathbf{y}_{i}
$$


If $\hat{\mathbf{x}}_{\mathbf{i}}(\lambda)$ is a good estimate for $\mathbf{x}$, then $\mathbf{h}_{\mathbf{i}} \hat{\mathbf{x}}_{\mathbf{i}}(\lambda)$ should be a good estimate of $\boldsymbol{h}_{\mathbf{i}} \mathbf{x}$. We don't have this term available, but an unbitsed estimate of it is $y_{i}$. Following this line of reasoning, we might wish to pick $\lambda$ to minimize $\left[y_{i}-\mathbf{h}_{\mathbf{i}} \hat{\mathbf{x}}_{\mathbf{i}}(\lambda)\right]^{2}$. Of course, we shouldn't favor any particular row, so we pick $\lambda$ to minimize the average error over all $i$; i.e., we pick the value of $\lambda$ that minimizes

$$
P(\lambda)=\frac{1}{n} \sum_{i=1}^{n}\left[y_{i}-\mathbf{h}_{i} \hat{\mathbf{x}}_{i}(\lambda)\right]^{2}
$$

This is the usual cross validation statistic for our problem and is sometimes called Allen's PRESS [15].

Minimizing Eq. (35) is a prediction error method that has a lot of intuitive appeal. Unfortunately, however, this method is not completely satisfactory. In the case where both $\mathbf{H}$ and $\mathbf{F}$ are identity matrices, $P(\lambda)=\frac{1}{n} \sum_{i=1}^{n} y_{i}^{2}$, and there is no defined minimum. In addition, this method is not rotation invariant. Suppose that instead of observing $\mathbf{y}$ we observe $\tilde{\mathbf{y}}=\mathbf{R y}$, where $\mathbf{R}^{T} \mathbf{R}=\mathbf{I}$, i.e., $\mathbf{R}$ is an orthogonal matrix. The model then becomes, instead of Eq. (3)

$$
\mathbf{R H x}+\mathbf{R e}=\tilde{\mathbf{y}} .
$$

This change of model is only an orthogonal change of coordinate system, and it is easy to see that the noise for this model, Re, has the same statistics as e. Therefore, one would expect that applying cross validation to the rotated model would yield the same estimate for $\mathbf{x}$. Unfortunately, such is not the case; the minimizing values of $\lambda$ are usually different. GCV is a form of cross validation that is independent of rotation of the coordinate system. In particular the model is transformed into one which is independent of the original coordinate system, then Eq. (35) is applied to that model. The singular value decomposition of $\mathbf{H}$ is

$$
\mathbf{H}=\mathbf{U D V}^{T}
$$

Where $\mathbf{U}$ is $n \times n$ orthogonal, $\mathbf{V}$ is $p \times p$ orthogonal, and $\mathbf{D}$ is $n \times p$ and nonzero only on its principal diagonal. Observe that if $\mathbf{R}$ is orthogonal, then the singular value 
decomposition of $\mathbf{R H}$ is

$$
\mathbf{R H}=\mathbf{R U D V}^{T}
$$

therefore, the model

$$
\begin{aligned}
\tilde{\mathbf{y}} & =\mathbf{U}^{T} \mathbf{y}+\mathbf{U}^{T} \mathbf{e} \\
& =\mathbf{D V}^{T} \mathbf{x}+\tilde{\mathbf{e}}
\end{aligned}
$$

is independent of any rotations of the coordinate system. We next premultiply this model by the unitary (i.e., $\mathbf{W}^{H} \mathbf{W}=\mathbf{I}$, where $\mathbf{W}^{H}$ is the conjugate transpose of $\mathbf{W}$ ) matrix

$$
\mathbf{W}=\frac{1}{\sqrt{n}}\left[e^{\frac{2 \pi i k l}{n}}\right]_{k l},
$$

to give the model

$$
\tilde{\tilde{\mathbf{y}}}=\mathrm{WDV}^{T} \mathbf{x}+\mathbf{W} \tilde{\mathbf{e}}
$$

to which we apply usual cross validation method given by Eq. (35). Note that this model is also rotation independent, i.e., it will be the same regardless of whether we began with H or RH: After a large amount of algebra, which we omit, it can be shown that Eq. (35) when applied to this model becomes

$$
V(\lambda)=\frac{\frac{1}{n} \operatorname{RSS}(\lambda)}{\left[\frac{1}{n} \operatorname{Trace}\{\mathbf{I}-\mathbf{H L}(\lambda)\}\right]^{2}} .
$$

It is also possible to show, after more algebraic pain that we omit, that GCV may be viewed as a weighted version of ordinary cross validation:

$$
V(\lambda)=\frac{1}{n} \sum_{i=1}^{n}\left[y_{i}-\mathbf{h}_{i} \hat{\mathbf{x}}_{i}(\lambda)\right]^{2} w_{i i}(\lambda) .
$$

The weights are

$$
w_{i i}(\lambda)=\left(\frac{1-a_{i i}(\lambda)}{1-\frac{1}{n} \sum_{j=1}^{n} a_{j j}(\lambda)}\right)^{2}
$$


where $a_{i i}(\lambda)$ is the $(i, i)^{t h}$ entry of the so-called influence matrix

$$
\begin{aligned}
\mathbf{A}(\lambda) & =\mathbf{H L}(\lambda) \\
& =\mathbf{H}\left(\mathbf{H}^{T} \mathbf{H}+\lambda \mathbf{F}^{T} \mathbf{F}\right)^{-1} \mathbf{H}^{T} .
\end{aligned}
$$

The term "influence matrix" comes from the fact that $\mathbf{A}(\lambda)$ measures the effect of the observed output $\mathbf{y}$, on the predicted output

$$
\begin{aligned}
\hat{\mathbf{y}}(\lambda) & =\mathbf{H} \hat{\mathbf{x}}(\lambda) \\
& =\mathbf{A}(\lambda) \mathbf{y}
\end{aligned}
$$

hence,

$$
\frac{\partial \hat{y}_{i}(\lambda)}{\partial y_{j}}=a_{i j}(\lambda)
$$

We can use the influence matrix to write both the ordinary cross validation function and the generalized cross validation function as weighted versions of residual sum of squares. After more algebra the regular cross validation function becomes

$$
P(\lambda)=\frac{1}{n} \sum_{i=1}^{n}\left[\frac{1}{1-a_{i i}(\lambda)}\right]^{2}\left[y_{i}-\hat{y}_{i}(\lambda)\right]^{2}
$$

and the generalized cross validation function becomes

$$
V(\lambda)=\frac{1}{n} \sum_{i=1}^{n}\left[\frac{1}{1-\frac{1}{n} \sum_{j=1}^{n} a_{j j}(\lambda)}\right]^{2}\left[y_{i}-\hat{y}_{i}(\lambda)\right]^{2} .
$$

We may interpret these two expressions is follows. The $i^{\text {th }}$ entry of the residual vector is $r_{i}(\lambda)=y_{i}-\hat{y}_{i}(\lambda)$, and

$$
\frac{\partial r_{i}(\lambda)}{\partial y_{i}}=1-a_{i i}(\lambda)
$$

Consequently, Eqs. (48) and (49) can be written as, respectively,

$$
P(\lambda)=\frac{1}{n} \sum_{i=1}^{n}\left[\frac{1}{\frac{\partial r_{i}(\lambda)}{\partial y_{i}}}\right]^{2}\left[r_{i}(\lambda)\right]^{2},
$$


and

$$
V(\lambda)=\frac{1}{n} \sum_{i=1}^{n}\left[\frac{1}{\frac{1}{n} \sum_{j=1}^{n} \frac{\partial r_{j}(\lambda)}{\partial y_{j}}}\right]^{2}\left[r_{i}(\lambda)\right]^{2} .
$$

In the case of regular cross validation, the $i^{\text {th }}$ residual, is weighted by the inverse of its sensitivity to changes in $y_{i}$. On the other hand, in the case of generalized cross validation, each residual is weighted by the same function. This function is the inverse of the average residual sensitivity.

It is also possible to provide a couple of arguments that show why generalized cross validation can be expected to work well under certain conditions. These arguments are developed in [13] for the case $\mathbf{F}=\mathbf{I}$, but they extend to the more general situation. The first argument is based on Bayesian reasoning. Suppose that $\mathbf{x}$ is a random vector that has the prior density given by Eq. (14), and our filter matrix is also $\mathbf{F}$. Then it is possible to show that the $\lambda$ that minimizes $E_{\mathbf{H}} E\{V(\lambda)\}$ is the same $\lambda$ that minimizes $E_{\mathbf{H}} E\{\hat{T}(\lambda)\}$. In particular, it is the $\lambda$ of Eq. (14). This allows us to conclude that, on the average, at least, the $\lambda$ 's obtained from methods three and four should be very close. The notation perhaps needs explaining. $E$ denotes expectation over the output noise $\mathbf{e}$ with $\mathbf{x}$ fixed, and $E_{\mathbf{H}}$ denotes expectation over the unknown $\mathbf{x}$ according to the prior given by $\mathrm{Eq}$. (14). The second argument does not assume any kind of prior distribution on the unknown, and also shows that under certain conditions the $\lambda$ 's selected by methods three and four will have similar values. In particular:

$$
\frac{\left|E\{\hat{T}(\lambda)\}-E\{V(\lambda)\}+\sigma^{2}\right|}{E\{\hat{T}(\lambda)\}}<\left(2 \mu_{1}+\frac{\mu_{1}^{2}}{\mu_{2}}\right) \frac{1}{\left(1-\mu_{1}\right)^{2}}
$$

where

$$
\mu \boldsymbol{1}=\frac{1}{n} \operatorname{Trace}\{\mathbf{A}(\lambda)\}
$$

and

$$
\mu_{2}=\frac{1}{n} \operatorname{Trace}\left\{\mathbf{A}^{2}(\lambda)\right\}
$$

So that if both $\mu_{1}$ and $\frac{\mu_{1}^{2}}{\mu_{2}}$ are small, then $E\{V(\lambda)\} \approx E\{\hat{T}(\lambda)\}+\sigma^{2}$. Consequently, on the average the minimizer of $V(\lambda)$ will be close to the minimizer of $\hat{T}(\lambda)$. 
Let $\nu_{1}, \ldots, \nu_{p}$ be the $p$ singular values of $\mathbf{H}$, and suppose that $\mathbf{F}=\mathbf{I}$. Then

$$
\mu_{1}=\frac{1}{n} \sum_{j=1}^{p} \frac{\nu_{j}}{\nu_{j}+\lambda}
$$

and

$$
\mu_{2}=\frac{1}{n} \sum_{j=1}^{p}\left(\frac{\nu_{j}}{\nu_{j}+\lambda}\right)^{2} .
$$

It follows immediately that both $\mu_{1}$ and $\frac{\mu_{1}^{2}}{\mu_{2}}$ are less than $\frac{p}{n}$ so that GCV can be expected to wcrk well if the data records are considerably longer than the unknown. Similarly, GCV can be expected to work well if $\mathbf{H}$ has only $q \ll n$ significant singular values. This corresponds to the situation where the kernel $\{h(n)\}$ has a nearly zero response over much of the frequency spectrum. For many problems $\{h(n)\}$ is smooth and so is low pass. These arguments can also be extended to the case where $\mathbf{F}$ is not the identity matrix. In particular, they apply if $\{f(n)\}$ has a sufficiently large amount of energy in the parts of the spectrum where the response of $\{h(n)\}$ is small. In other words, if $\{h(n)\}$ is low pass and $\{f(n)\}$ is high pass, GCV can be expected to work well.

On the other hand, it is important to note that we have observed situations in which the performance of GCV was not satisfactory. However, these occurred when the conditions outlined above were violated. In particular the problem was well posed and "square" so that there were $q=n$ significant eigenvalues. This problem does not appear to have been considered in the literature. Our intuition is that GCV uses that portion of the output space where there is no signal (because $\{h(n)\}$ does not have a response in that portion) to estimate the noise variance, so GCV might be expected to perform poorly when the problem is well posed.

\section{Nonnegative Estimates}

The methods described above may also be used when $\hat{\mathbf{x}}$ is constrained to be nonnegative, but some modifications are necessary. Define the index sets $\mathcal{P}$ and $\mathcal{Z}$ to be such that $\mathcal{P} \cup \mathcal{Z}=\{1,2, \ldots, p\}$, and $\mathcal{P} \cap \mathcal{Z}=\emptyset$. The notation $\mathbf{H}_{\mathcal{P}}$ will be used to denote the matrix formed from $\mathbf{H}$ by retaining only the columns corresponding to $\mathcal{P}$; similarly, the 
notation $\mathbf{x}_{\mathcal{P}}$ denotes the vector formed from $\mathbf{x}$ by keeping unly those entries corresponding to $\mathcal{P}$. For a given $\mathcal{P}$, define the vector $\tilde{\mathbf{x}} \mathcal{P}(\lambda)$ to be the solution to the unconstrained least squares problem

$$
\left[\begin{array}{r}
\mathbf{H}_{\mathcal{P}} \\
\sqrt{\lambda} \mathbf{F}_{\mathcal{P}}
\end{array}\right] \tilde{\mathbf{x}}_{\mathcal{P}} \quad \begin{aligned}
& \text { l.s. } \\
&
\end{aligned}\left[\begin{array}{l}
\mathbf{y} \\
\mathbf{0}
\end{array}\right] .
$$

From Eqs. (12) and (13), we know that the solution to Eq. (55) is

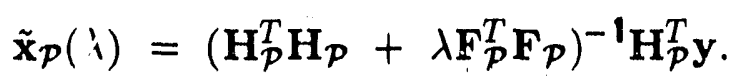

The form of the solution to a linear least squares problems that has nonnegativity constraints on the unknown is derived using the Kuhn-Tucker conditions in [16]. Among all of the $2^{p}$ possible index sets in Eq. (56), consider only those for which $\tilde{\mathbf{x}} \boldsymbol{p}(\lambda)>0$, and select the one that minimizes the squared error in Eq. (55); call this particular set $\mathcal{P}(\lambda)$. Then the solution to Eq. (16) is the vector $\hat{\mathbf{x}}^{+}(\lambda)$ where

$$
\begin{aligned}
& \hat{\mathbf{x}}_{\mathcal{P}(\lambda)}^{+}(\lambda)=\tilde{\mathbf{x}}_{\mathcal{P}(\lambda)}(\lambda) \\
& \hat{\mathbf{x}}_{\mathcal{Z}(\lambda)}^{+}(\lambda)=\mathbf{0} .
\end{aligned}
$$

In other words, the way to find the solution to Eq. (16) is to try all possible subsets of variables in (55) and select the solution that gives the best fit and is positive; the remaining variables are set to zero. We note as an aside that one would never actually try all possible subsets; there are very efficient algorithms that at each iteration change subsets in such a way that the least squares fit is always improved. The code uses the NNLS algorithm described in [16].

As in the unconstrained case, the residual energy increases as $\lambda$ increases, but the energy in the solution decreases. Methods one and two are both "common sense" approaches that do not involve evaluating statistical quantities such as expectations and covariance matrices. Therefore, the approach to selecting the best $\lambda$ for methods one and two for the nonnegativity case is exactly the same as for the unconstrained case. We have observed that the discrepancy principle appears to exhibit about the same behavior for the 
constrained case. Unfortunately, however, the situation becomes more complicated in the case of $C_{L}(\lambda)$ and GCV. This is because the constraints on the solution cause distributions (i.e. delta functions) to occur in the probability density functions of the estimates. This makes the derivation of a precise prediction error statistic of the form shown in Eq. (31) all but impossible. A good intuitive discussion of how to deal with estimates that are subject to inequality constraints is given in Section 7.7 of [1]. The bottom line is that approximations are necessary, and that is the approach we take here.

If we retrase the arguments for $C_{L}(\lambda)$ and GCV, we see that Eqs. (31) and (42) can be used to judge the quality of a more general set of estimators than those of the form $\hat{\mathbf{x}}(\lambda)=\mathbf{L}(\lambda) \mathbf{y}$ defined by Eq. (21) above. In fact, consider any set of estimators of

the form $\hat{\mathbf{x}}(\theta)=\tilde{\mathbf{L}}(\theta) \mathbf{y}$, where $\tilde{\mathbf{L}}(\theta)$ is an arbitrary set of matrices parameterized by $\theta$. Then Eq. (31) or Eq. (42) can be used to select the $\theta$ corresponding to the best $\tilde{\mathrm{L}}(\theta)$. For example, in the unconstrained case, it is possible to use these equations to select the best kind of smoothing as well as the best $\lambda$ for that smoothing. We considered using $C_{L}$ to select the best kind of smoothing in [10]; the use of GCV to the select the type of smoothing is considered in [17]. For the nonnegativity constraint problem, we define $\mathbf{L}^{+}(\lambda)$ as follows: if $j \in \mathcal{P}(\lambda)$, then the $j^{\text {th }}$ row of $\mathbf{L}^{+}(\lambda)$ is the corresponding row of $\left(\mathbf{H}_{\mathcal{P}(\lambda)}^{T} \mathbf{H}_{\mathcal{P}(\lambda)}+\lambda \mathbf{F}_{\mathcal{P}(\lambda)}^{T} \mathbf{F}_{\mathcal{P}(\lambda)}\right)^{-1} \mathbf{H}_{\mathcal{P}(\lambda)}^{T}$; otherwise, if $j \in \mathcal{Z}(\lambda)$, then the $j^{\text {th }}$ row of $\mathbf{L}^{+}(\lambda)$ is zero. This corresponds to the estimates given by Eq. (57). The problem with this approach is that $\mathcal{P}(\lambda)$ depends on the observed output $\mathbf{y}$, and we do not take expectation over $\mathbf{y}$ to determine some kind of "average" $\mathcal{P}(\lambda)$ because the problem is too complicated. This approximate approach to $C_{L}$ and GCV works very well and is computationally simple. A detailed analysis of this approach, extensions to more accurate estimates of prediction error in the presence of inequality constraints, and more efficient computational methods for all of these methods will be the subjects of future journal articles.

\section{Numerical Issues and Portability}

In developing the deconvolution package we tried to use standard readily available 
numerical software whenever possible. Subroutines from LINPACK [18] were used to solve most matrix and vector problems. One exception was the nonnegatively constrained case where we used the NNLS algorithm [16]. We modified NNLS siightly to return the number of positive variables; this was required to compute the approximate $C_{L}$ and GCV statistics. A one variable zero finding algorithm was required for the discrepancy principle and the constrained energy methods, and a one variable minimization algorithm was required for $C_{L}$ and GCV. We used versions of the ZEROIN and FMIN algorithms described in [19].

Machine dependent numbers such as $\mathrm{i} / \mathrm{o}$ unit numbers and precisions are obtained from the subroutines IMACH and DMACH. The user should change these routines so that they correspond to his particular computer. Data files are read in and out by the subroutines GETDAT and PUTFIL. In the argument list of each of these subroutines is the variable IFM. If IFM is zero, the first line of a file will be a format statement so that the file will be compatible with SIG [20]. If IFM is one, the format statement is not included.

Our method for finding the minimum of the $C_{L}$ or the GCV statistic could use some additional work. Currently we use the subroutine FINDIT to bracket a minimum, and then FMIN searches for the minimum in the interval determined by FINDIT. Unfortunately, as noted in Ref. [21], the GCV function may have local minima, and a better approach would be to first perform a rough global search, and then apply FMIN to find an exact minimum. We noted in Ref. [10] that we did not see local minima in the $C_{L}$ statistic, but our guess is that although the $C_{L}$ statistic is better behaved than the GCV statistic, there are cases where local minima exist. Pending improvements in the search method, if the user is not satisfied with his answer, he may wish to try a different starting interval for FINDIT to see if a different minimum can be determined.

\section{Examples}

In this section we will consider a deconvolution problem that raises most of the issues discussed above. The true input is the nonnegative signal shown in Fig. 2. It is 35 samples long and consists of a square pulse 10 samples wide and a ramp five samples wide. The 
system impulse response is 41 samples long and is the smooth, lowpass function shown in Fig. 3. Convolving this function with the input smears the input's sharp transitions and destroys most of its high frequency content. The noise-free output signal that results is $35+41-1=75$ samples long and is shown in Fig. 4. The zero-mean, white, Gaussian noise signal shown in Fig. 5 has a variance of .007 and is added to the noise-free output to give the observed output data.

The purpose of our first set of examples is to demonstrate the superiority of timedomain methods over frequency-domain methods with respect to both variance and bias. One of the most common approaches to frequency-domain deconvolution/identification is to divide Fourier transforms and then low pass filter the resulting estimate. This may work well if any ill-posedness is due to the kernel's small response at high frequencies. However, if the kernel has spectral zeros elsewhere, then this method will yield a poor estimate. A better approach is to use a frequency-domain implementation of the constrained leastsquares approach we have taken in this paper. This approach is discussed in Refs. [22-24]. By replacing the Hankel matrix in Eq. (2) with a circulant approximation with each of its rows a zero padded "wrapped-around" version of $\{h(n)\}$, we obtain a circular convolution relationship that can be solved using the discrete Fourier transform (DFT). In the frequency domain the form of the estimate of $\{x(n)\}$ is

$$
\hat{X}(u)=\frac{H^{*}(u) Y(u)}{P\left(|H(u)|^{2}+\lambda|F(u)|^{2}\right)}, \quad u=0,1, \ldots, P-1,
$$

where $P$ is the order of the DFT. Applying Fourier- or Z-transform techniques to an estimator of the form of Eq. (58) would in general yield an estimate of $\{x(n)\}$ that has infinite length; therefore, using the DFT causes aliasing problems that must be considered. Writing Eq. (58) in the time domain and using the analysis in Ref. [24] we see that at the very least $P$ should be selected so that there is enough zero padding to have the convolutions on both sides of

$$
P\{x(n)\} \star[\{h(n)\} \star\{h(-n)\}+\{f(n)\} \star\{f(-n)\}]=\{h(-n)\} \star\{y(n)\}
$$


be linear, rather than circular. Consequently, if the length of the observed output record is $K$, the length of the smoothing kernel is $L$, the length of the unknown input is $M$, and the length of the system impulse response is $N$, then $P$ must satisfy:

$$
P \geq \max \{M+2 N-2, M+2 L-2, K+N-1\} .
$$

If we use zeroth-order smoothing for our example, the smallest possible value of $P$ is 115; furthermore, in order to use the fast Fourier transform ( FFT), we would probably choose a value of 128 .

The length of the resulting estimate should make the potential user more than a little nervous. There are only 75 noisy observations, yet we are estimating a sequence with 128 unknown parameters. This is a violation of the "parsimony principle" that says a systern should be modeled with enough parameters to describe its behavior, but no more [25]. In particular, these extra parameters will fit the noise, and we can expect a time-domain estimate that assumes an unknown sequence of length 35 to have lower variance. We demonstrate this behavior with the estimates shown in Figs. 6 and 7. Fig. 6 shows a time-domain estimate using zeroth order smoothing and GCV used to select $\lambda$. Fig. 7 shows a frequency-domain estimate with $P=128$ and $\lambda$ selected to minimize the squared estimation error:

$$
S=\sum_{i=1}^{35}[x(i)-\hat{x}(i)]^{2} .
$$

Even though we "cheated" by selecting $\lambda$ to minimize $S$, the time-domain estimate was superior: the time-domain estimate gave $S=1.79$, and the frequency-domain estimate gave $S=6.13$. The frequency-domain estimate for all 128 points is shown in Fig. 8 . The error in both of these estimates was caused by the output noise. In the noise-free case with $\lambda=0$, both time- and frequency-domain estimates are exact $(S=0)$ and are not shown. We speculate that for both time- and frequency-domain estimators in the noisy case the error is mostly variance, rather than bias.

However, our next example demonstrates that frequency-domain methods can under 
certain conditions yield severely biased estimates. In many transient experiments the data must be truncated before it has decayed to zero. For our example, pretend that the output data has been contaminated after the $60^{\text {th }}$ sample; consequently we wish to consider only the first 60 samples in obtaining an estimate. The first 60 samples of the noise-free output are shown in Fig. 9. The frequency-domain estimator zero pads this data, and the sharp jump to zero after the $60^{\text {th }}$ sample greatly biases the estimate. The noise-free frequencydomain estimate with $\lambda$ selected to minimize $S$ and $P=128$ is shown in Fig. 10. For this estimate, $S=12.9$. On the other hand, using a time-domain estimate with no postwindowing avoids the zero padding and gives $S=0$ for the noise-free case-there is no bias.

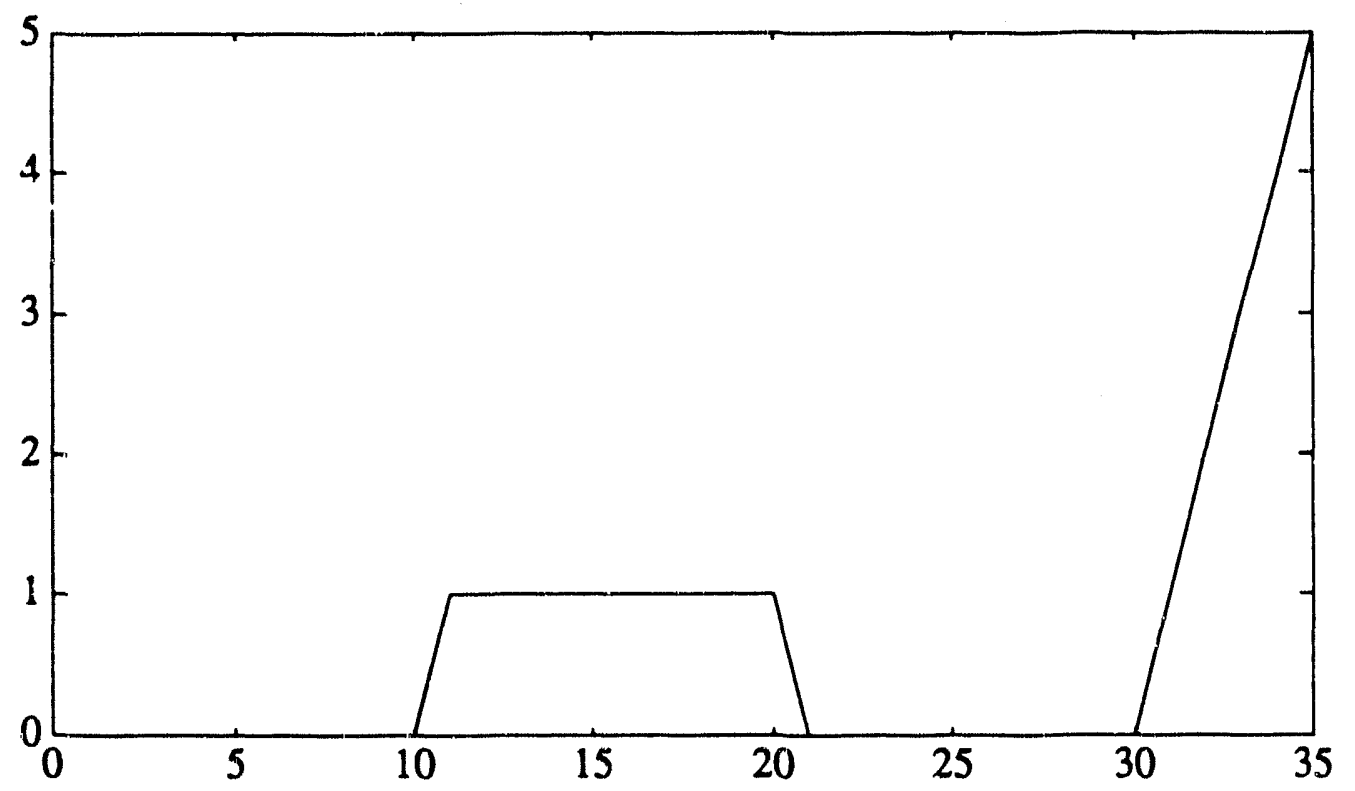

Fig. 2. Input Signal 


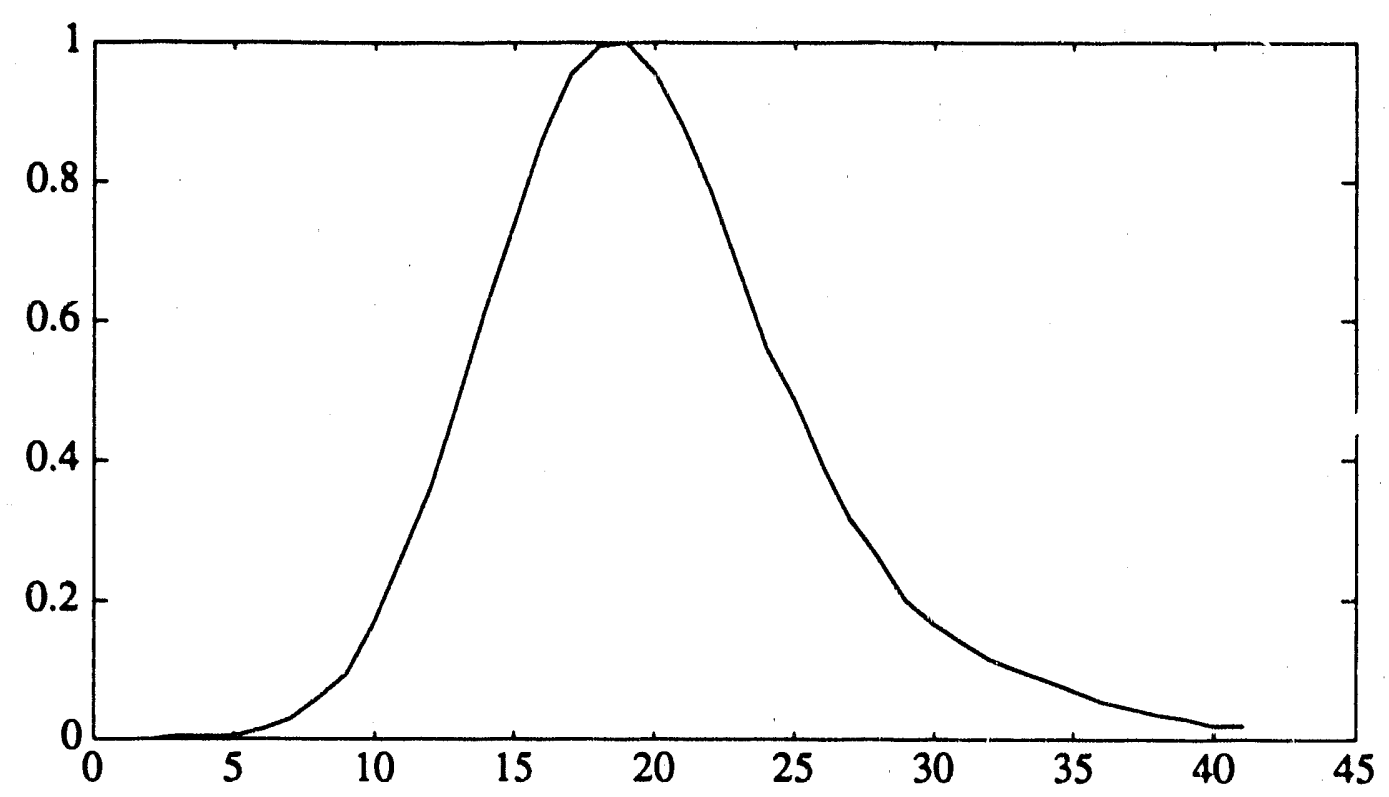

Fig. 3. System Impulse Response

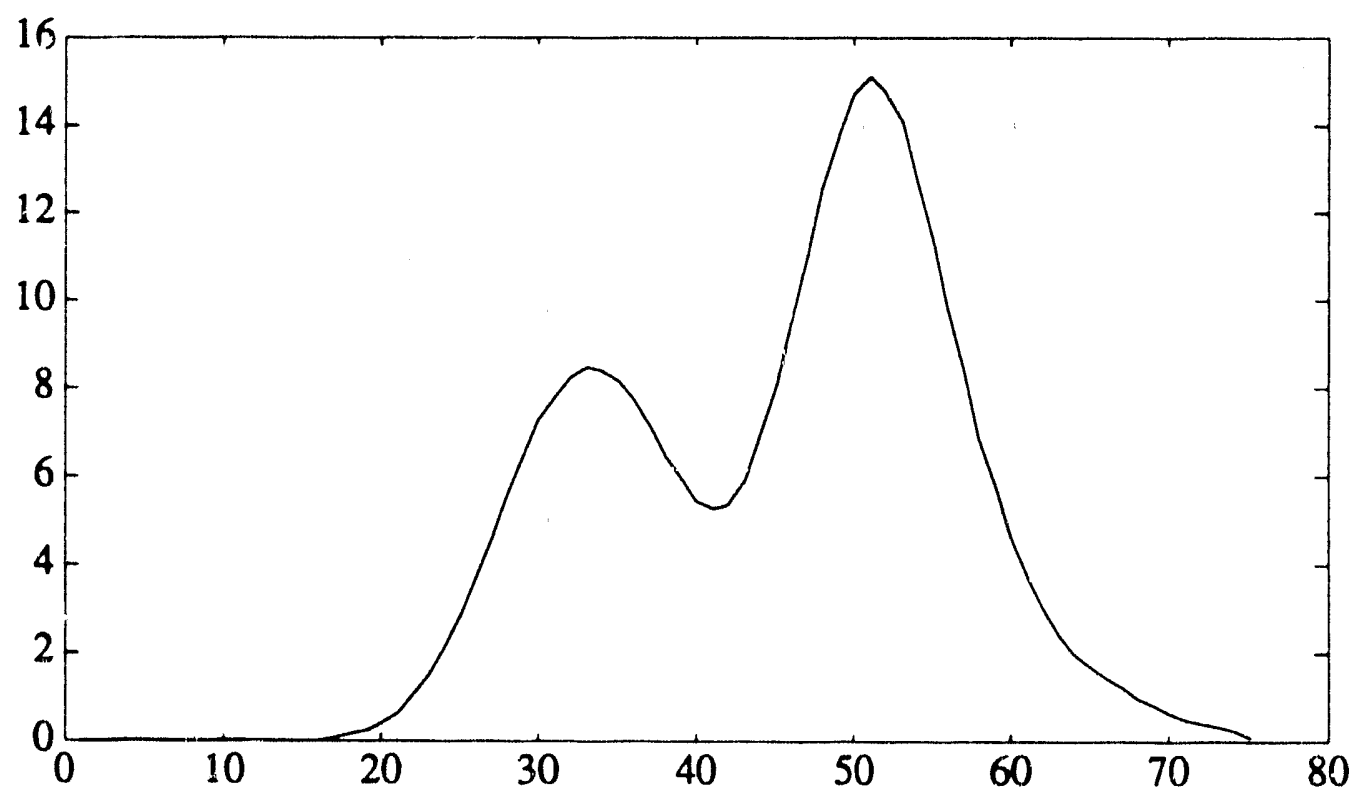

Fig. 4. Noise-Free Output 


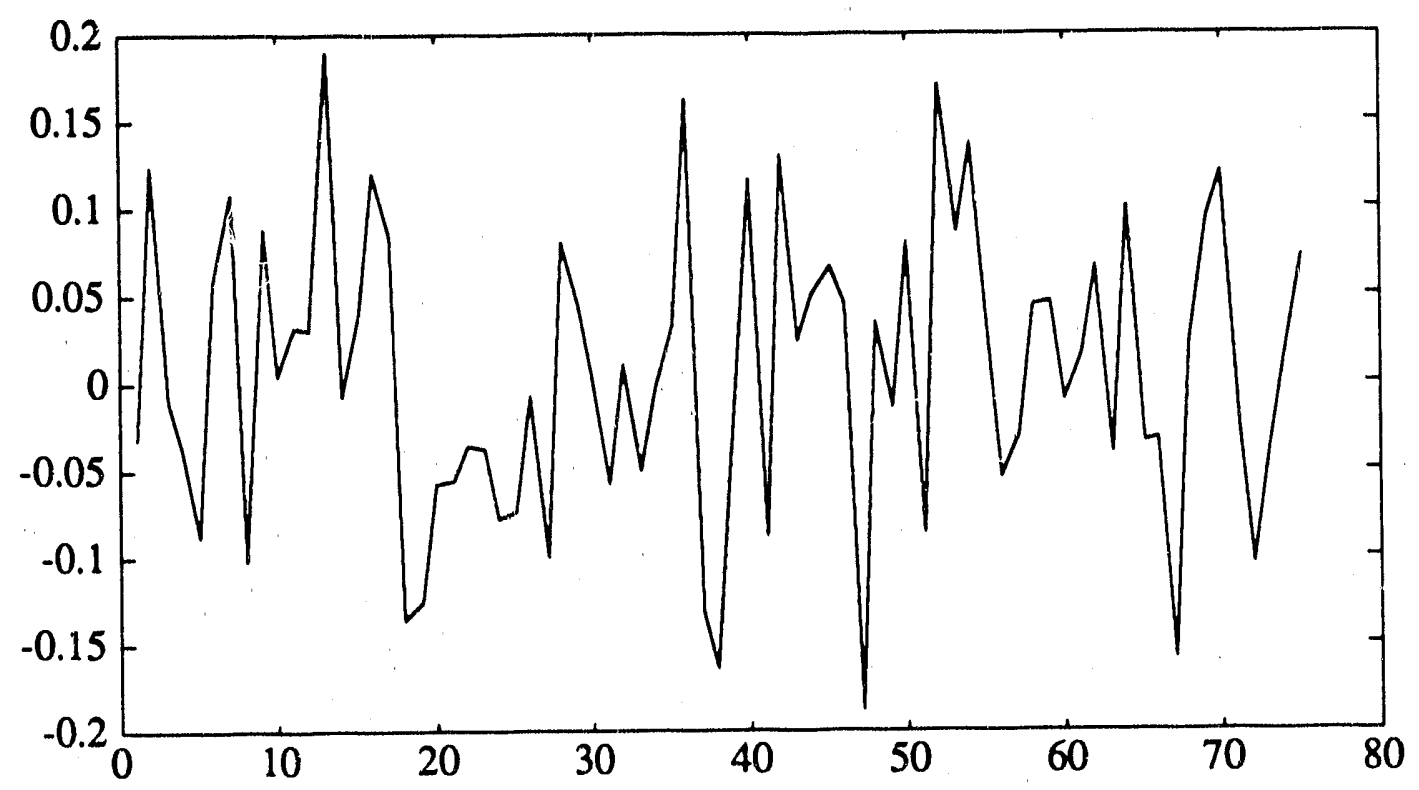

Fig. 5. Noise Signal

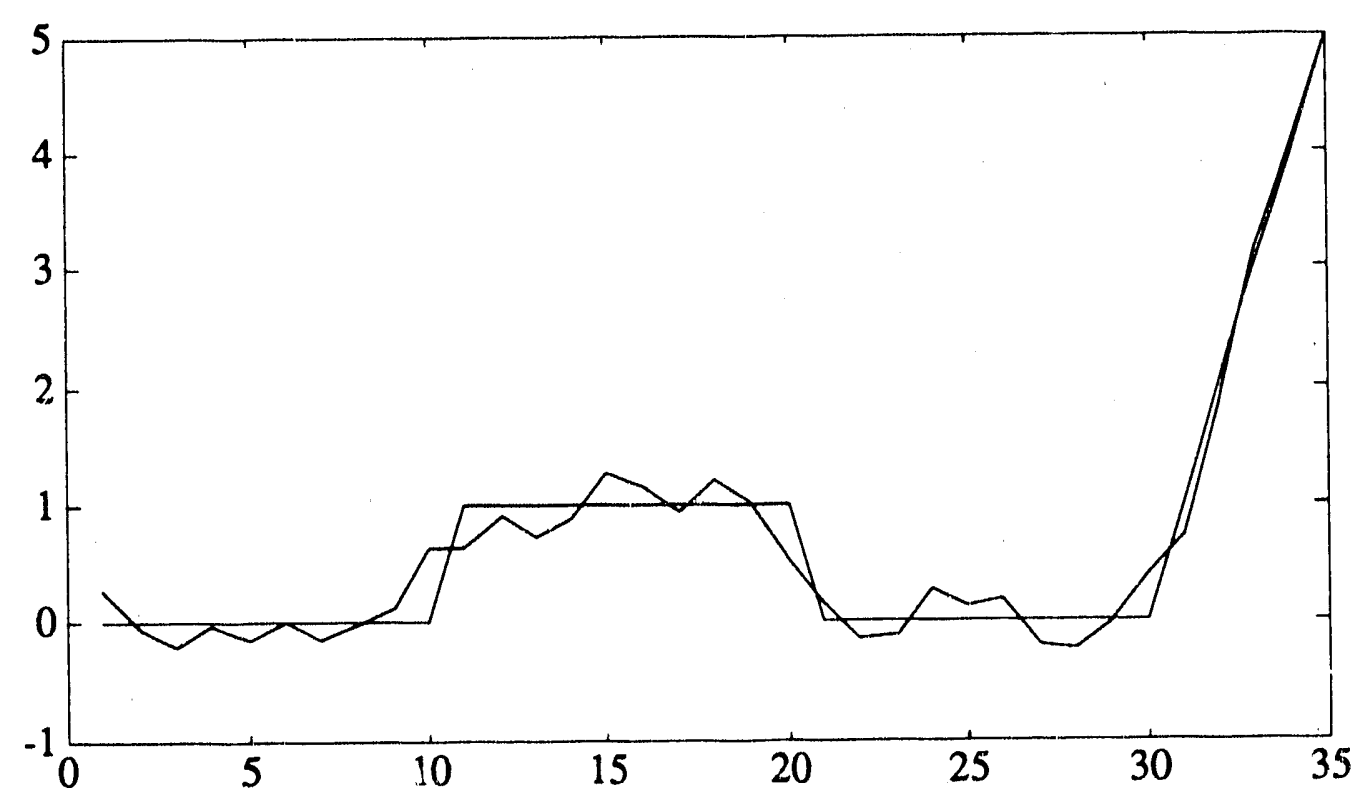

Fig. 6. Time-Domain Estimate 


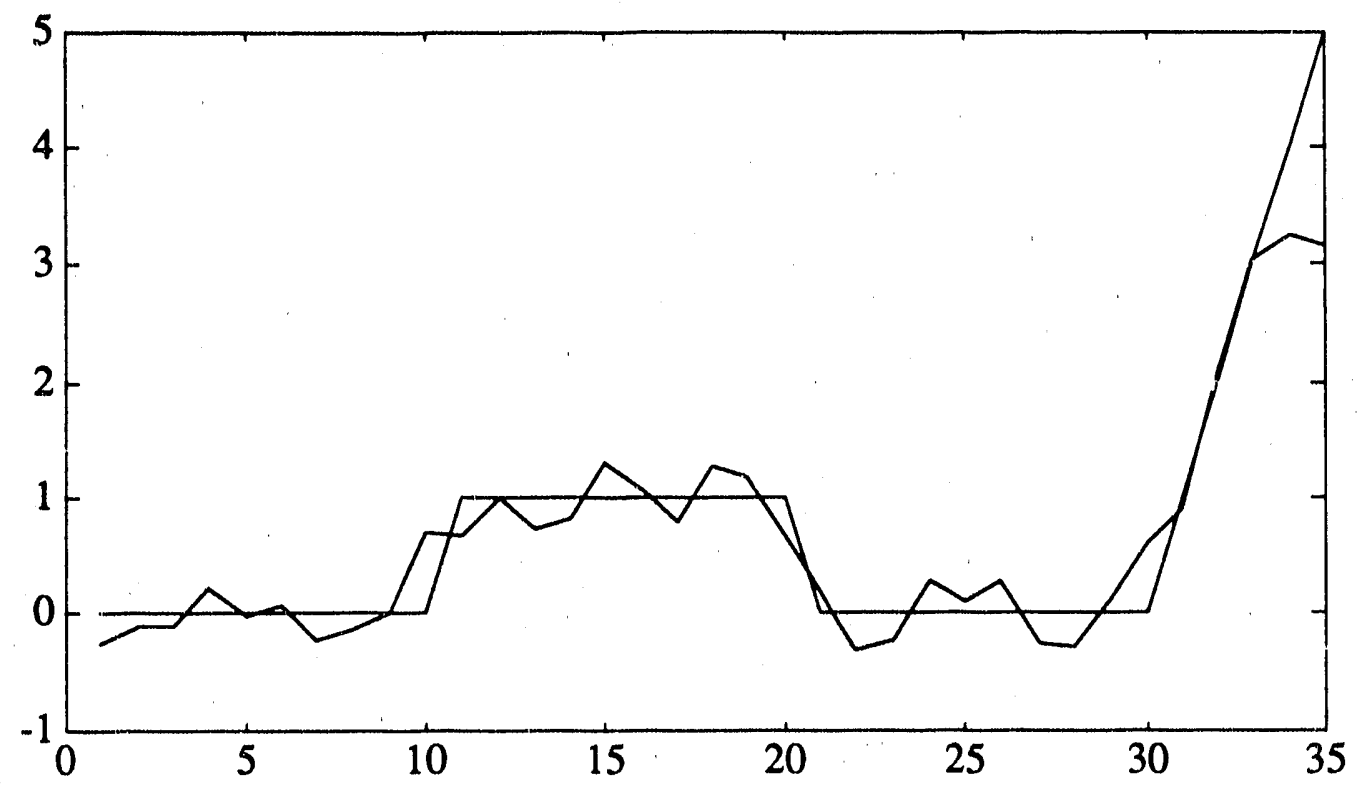

Fig. 7. Frequency-Domain Estimate

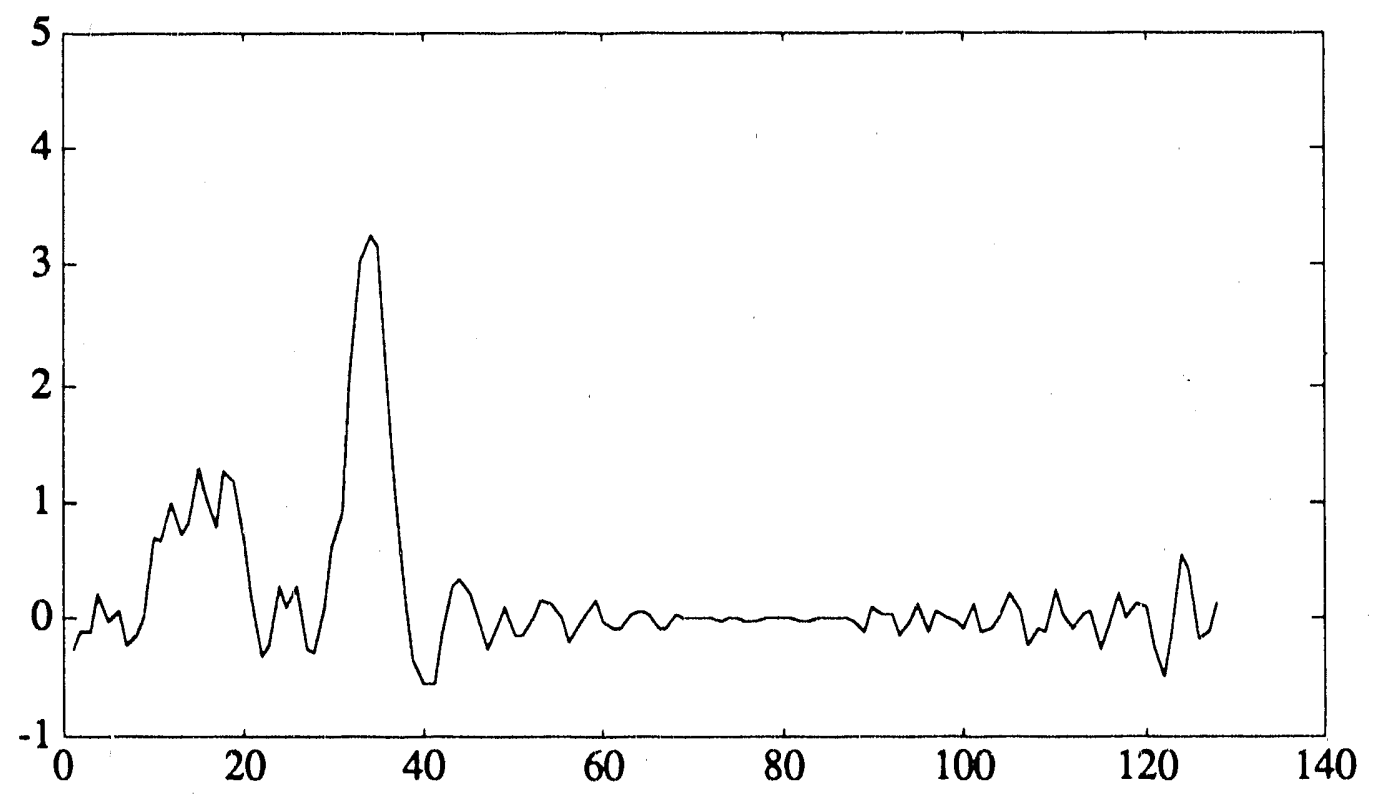

Fig. 8. Frequency-Domain Estimate 


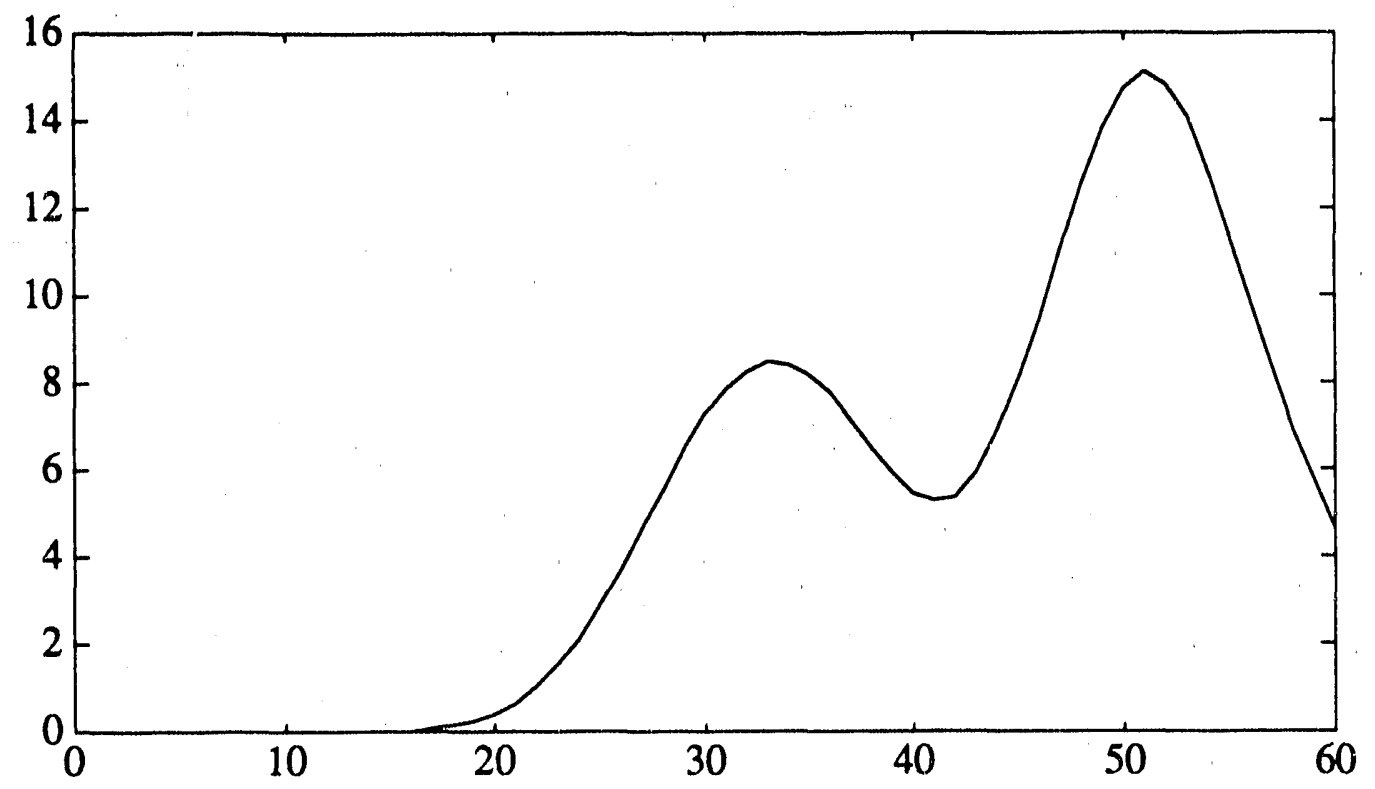

Fig. 9. Truncated Noise-Free Output

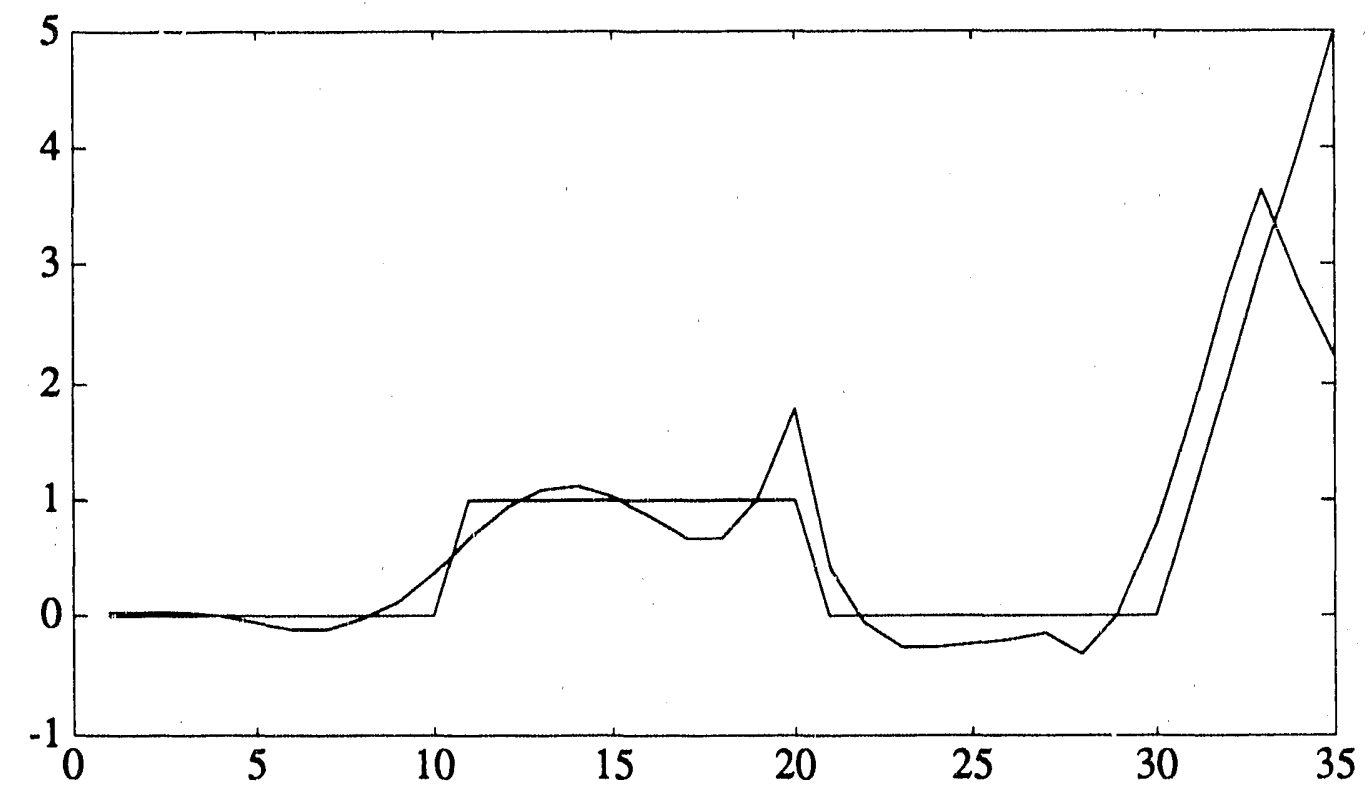

Fig. 10. Frequency-Domain Estimate for the Truncated Noise-Free Case 
We hope that the above examples have demonstrated the superiority of time-domain approaches. Although one of the tapering windows from time series analysis could be applied to bring the truncated output smoothly to zero before applying a frequency-domain method, the result is only a different-and possibly worse-kind of bias. The techniques that have been developed for determining transfer functions from long records of stationary random processes are in general not directly applicable to the transient problem. If data length prohibits direct matrix inversion techniques, there are other time-domain methods that are almost as fast as frequency-domain methods. The use of a Toeplitz, rather than a circulant, approximation to Eq. (2) permits fast inversion algorithms that do not force assumptions on the length of the unknown. However the data is still assumed to be zero padded. Iterative methods based on the conjugate gradient algorithm are almost as fast and do not force zero padding. They will be considered separately in a future report. A final comment concerning time vs. frequency domain is worthwhile. It follows immediately from Eq. (58) that a shift in either the kernel or the output will only cause a corresponding shift in the estimate. This is comforting but misleading: in particular, this is a circular shift. The time-domain estimate may change in other ways as the data is shifted, particularly if the data is not both pre- and post-windowed with the consequence that certain observed data are added or deleted with shifts.

Our next set of examples concentrates on the time domain. The data is the same as above; only the first 60 samples of the noisy output are used. To avoid truncation-induced bias the data is prewindowed but not postwindowed. Our first estimate demonstrates the importance of the correct decision on windowing the smoothing. For the estimate shown in Fig. 11 first-order smoothing is used, and the smoothing is both pre- and post-windowed. Postwindowing the smoothing assumes that the estimate is zero after the $35^{\text {th }}$ sample, and the smoothing tries to eliminate the sharp transition; hence, the large error at the end of the estimate. This example shows that if it is sispected that the unknown has not decayed to zero at the end of the estimation interval, the smoothing should not be postwindowed. 
This situation often arises in what we called the "typical experiment" in the Introduction. For Figs. 12-17 the smoothing is prewindowed but not postwindowed. Each of these figures presents a different way to select $\lambda$ with or without the nonnegativity constraint. The true input is represented by a solid line, the zeroth-order smoothed estimate by a dotted line, the first-order smoothed estimate by a dashed line, and the second-order smoothed estimate by a dot-dashed line. The results shown in Figs. 11-17 are summarized in Table 1. The first line of this table is for Fig. 11 where "1PSTW" stands for first-order smoothing with postwindowing.

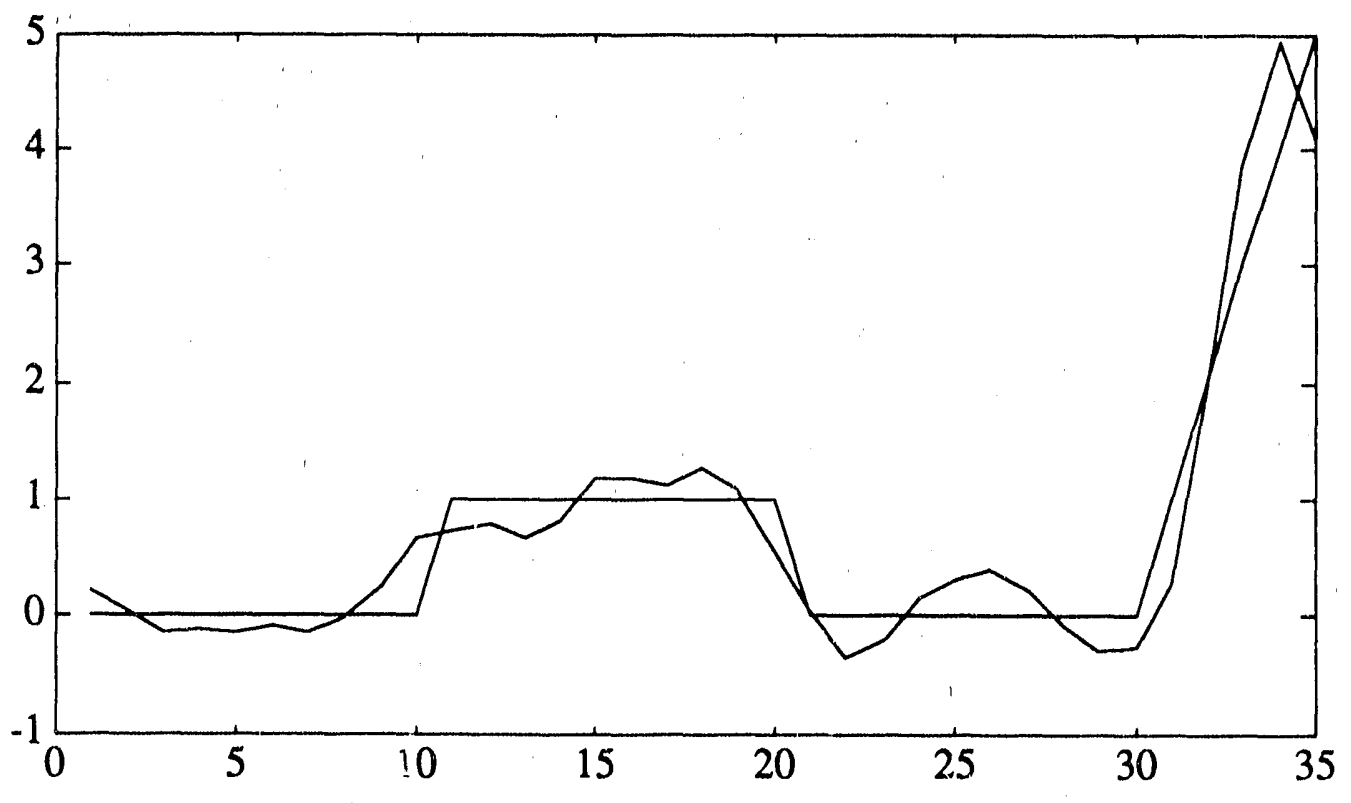

Fig. 11. Time-Domain Estimate with Postwindowed Smoothing 


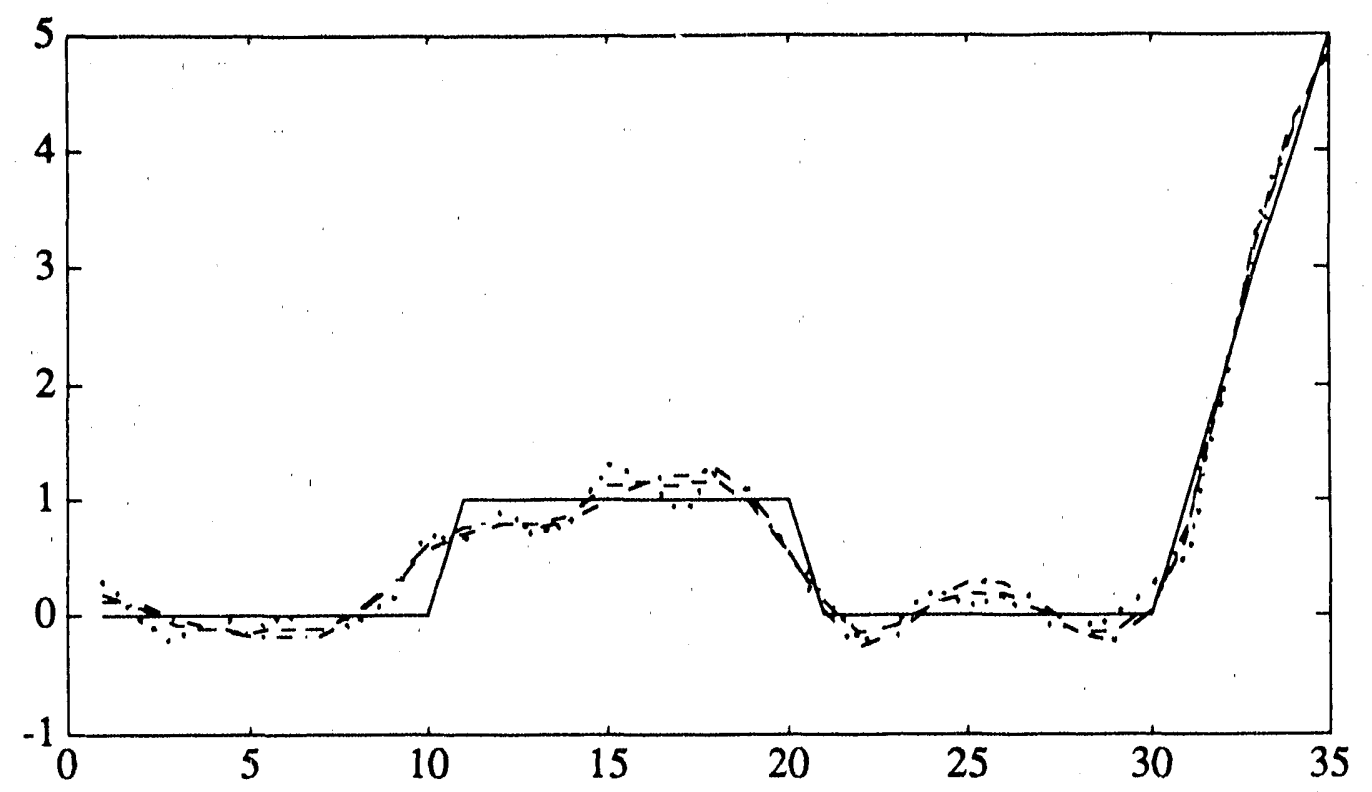

Fig. 12. Unconstrained GCV Estimates

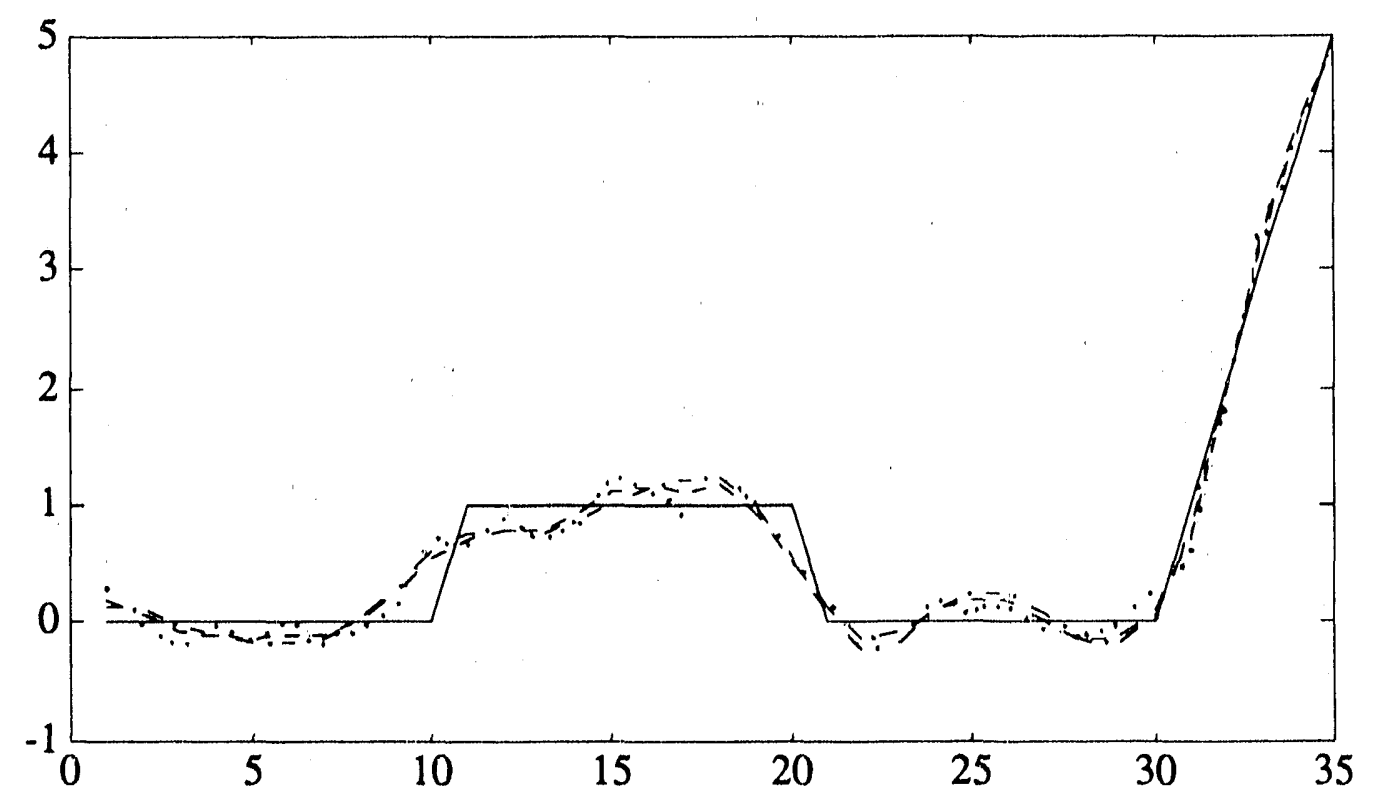

Fig. 13. Unconstrained Mallows' $C_{L}$ Estimates 


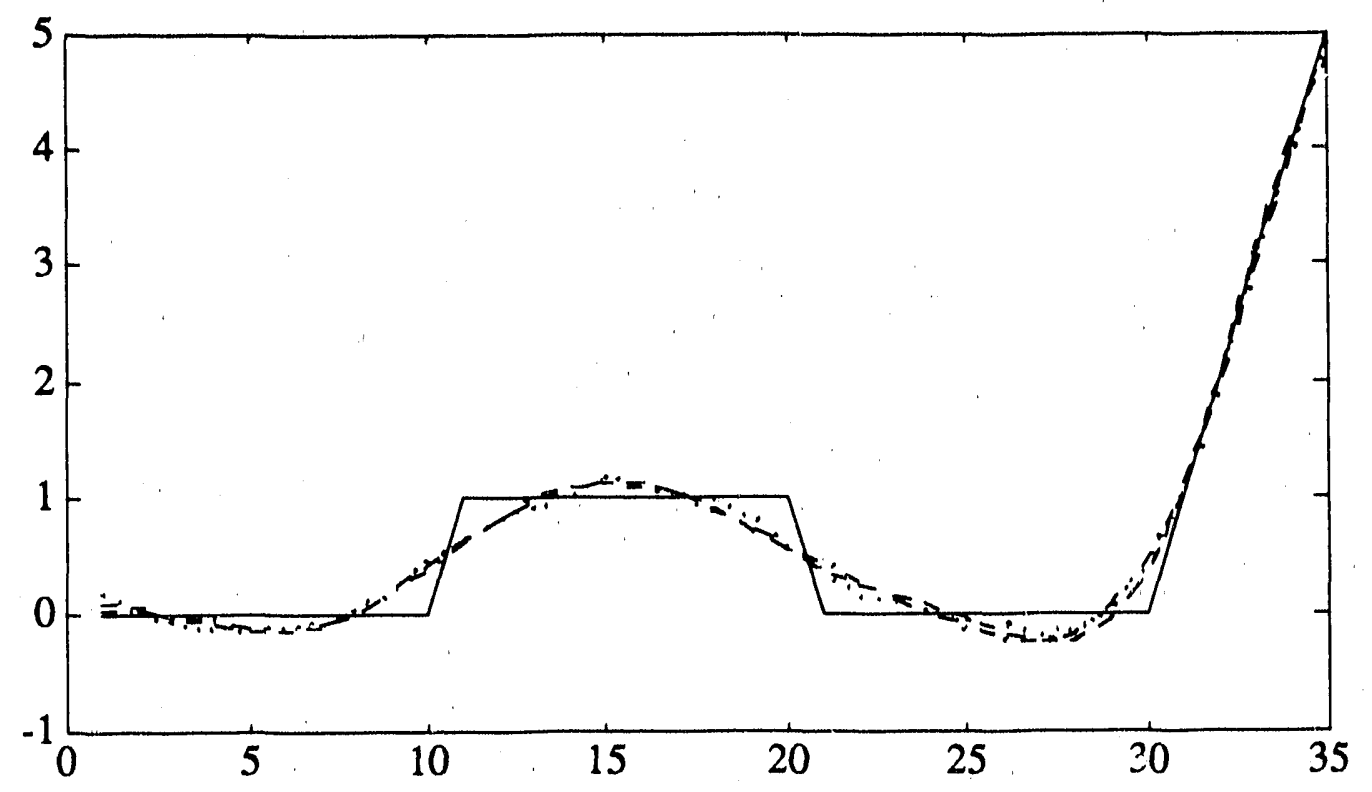

Fig. 14. Unconstrained Discrepancy Principle Estimates

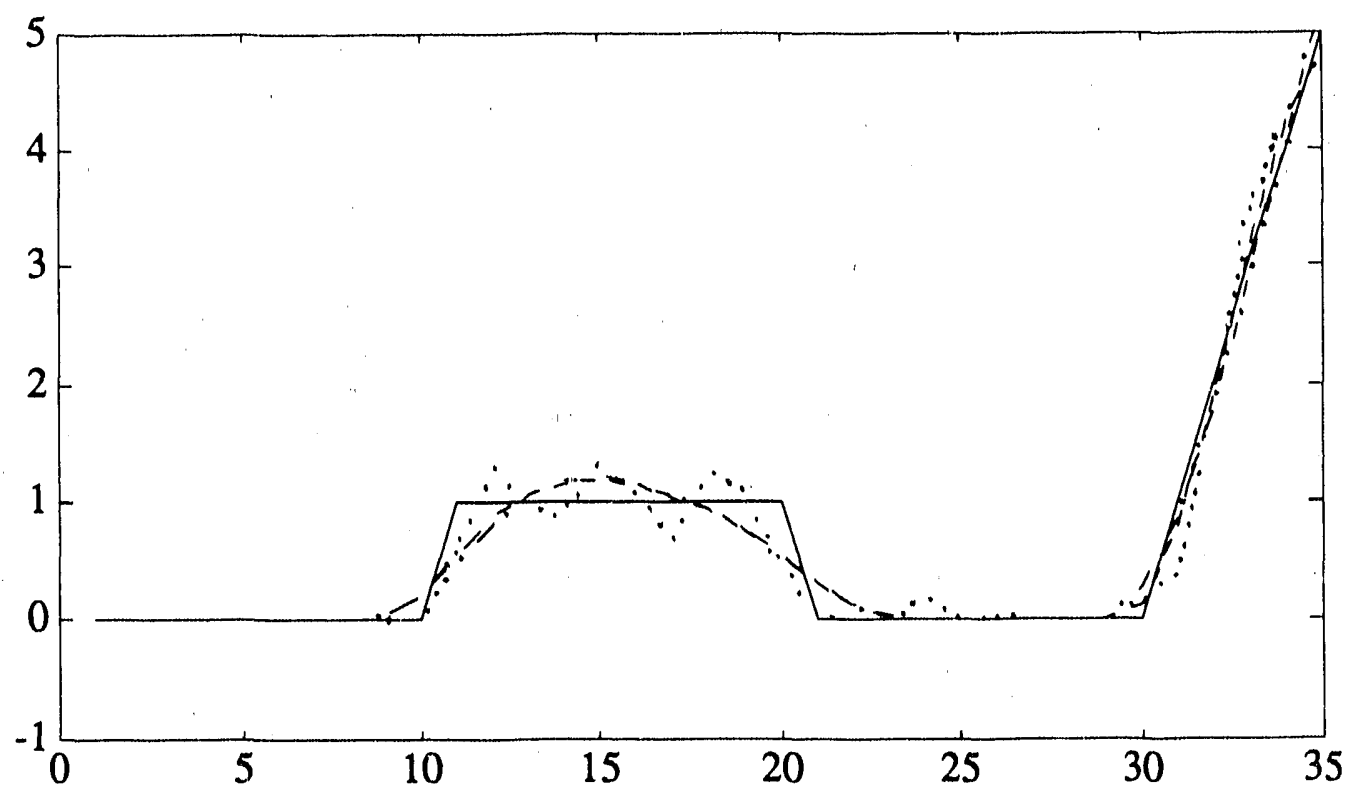

Fig. 15. Constrained GCV Estimates 


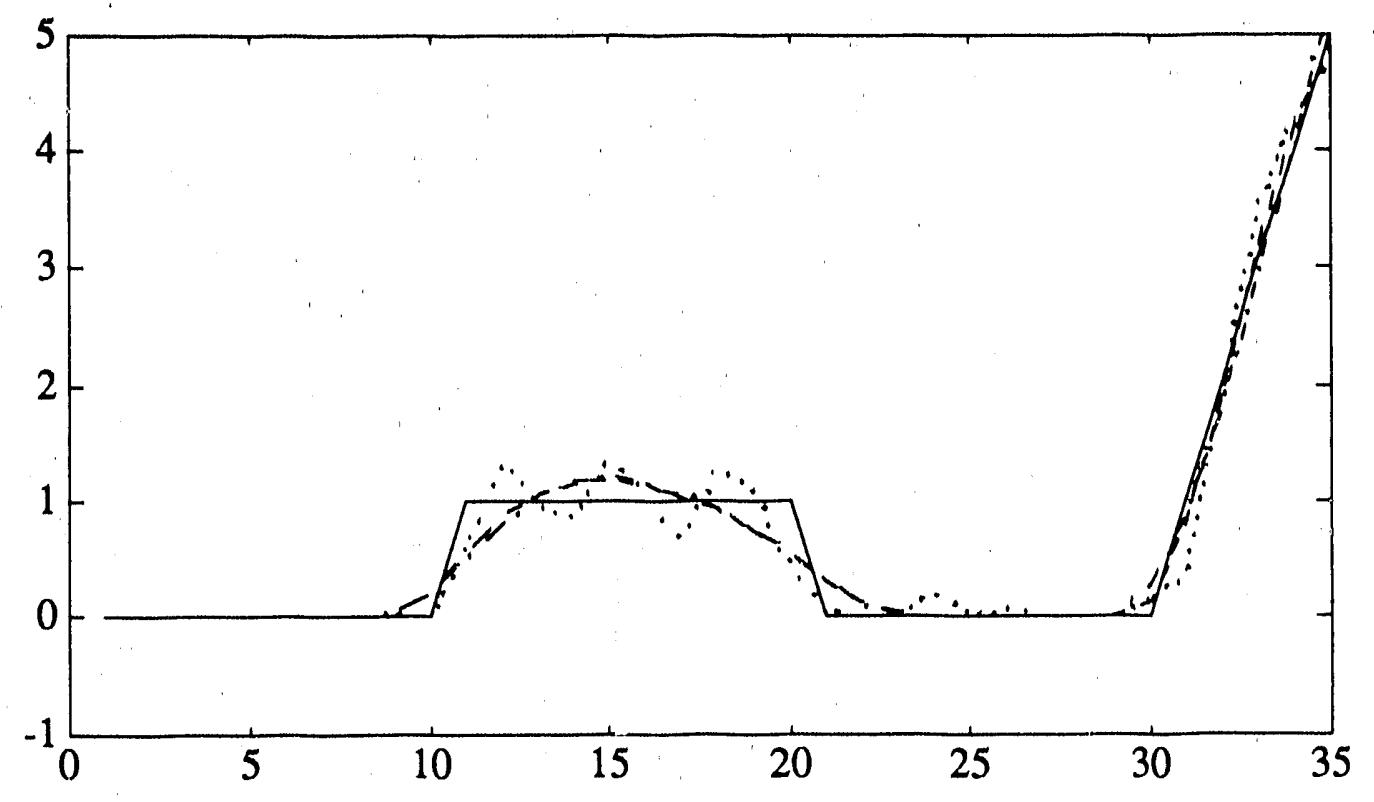

Fig. 16. Constrained Mallows' $C_{L}$ Estimates

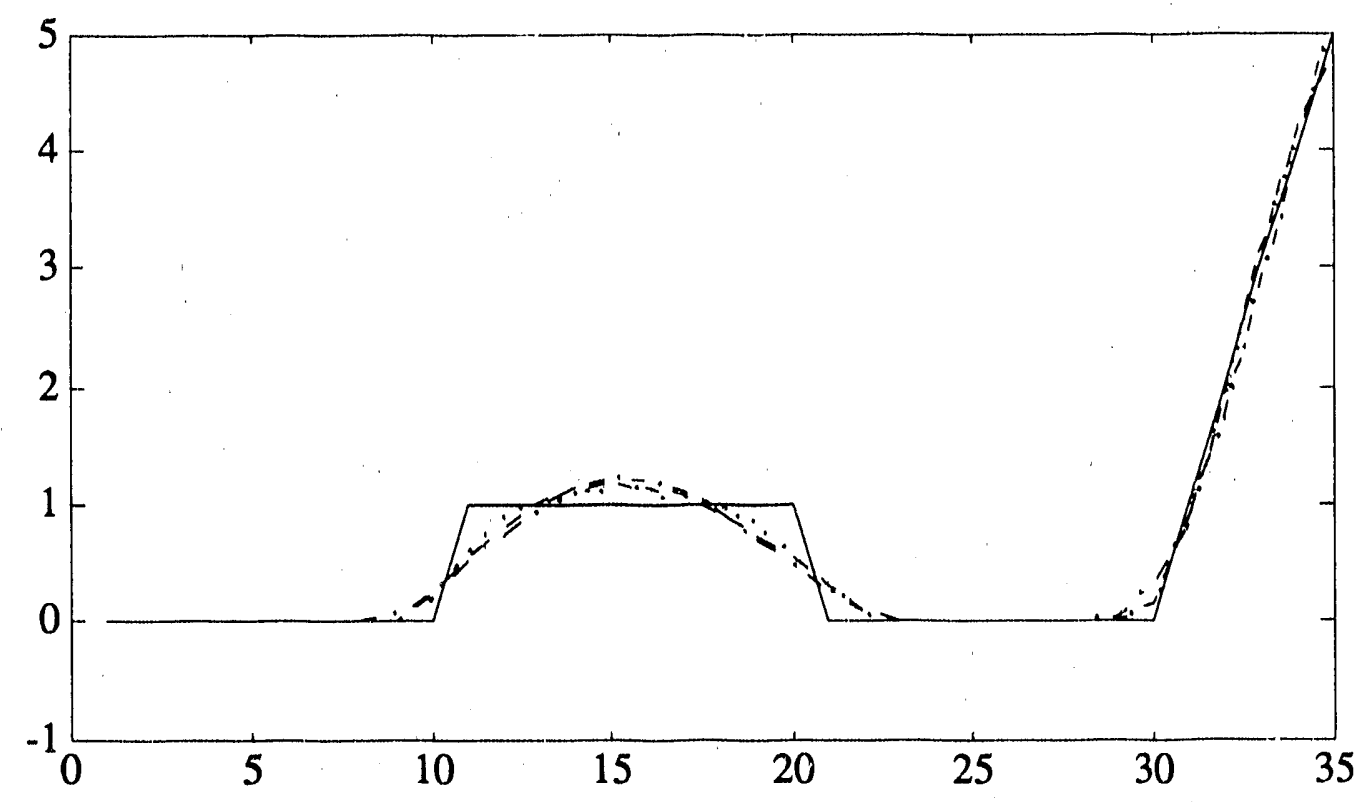

Fig. 17. Constrained Discrepancy Principle Estimates 


\begin{tabular}{|c|c|c|c|c|c|}
\hline Method & Constraint & Smoothing & Value of $\lambda$ & Residual MSE & Square Error $(S)$ \\
\hline $\mathrm{GCV}=.00806$ & NO & 1PSTW & .00847 & .00531 & 4.77 \\
\hline $\mathrm{GCV}=.00837$ & NO & 0 & .0104 & .00547 & 2.06 \\
\hline $\mathrm{GCV}=.00818$ & NO & 1 & .0166 & .00560 & 1.44 \\
\hline $\mathrm{GCV}=.00828$ & NO & 2 & .0128 & .00558 & 1.59 \\
\hline$C_{L}=9.87$ & NO & 0 & .0114 & .00551 & 1.92 \\
\hline$C_{L}=8.72$ & NO & 1 & .0184 & .00563 & 1.39 \\
\hline$C_{L}=9.32$ & NO & 2 & .0147 & .00561 & 1.50 \\
\hline D. P. & NO & 0 & .069 & .007 & 1.24 \\
\hline D. P. & NO & 1 & .186 & .007 & 1.36 \\
\hline D. P. & NO & 2 & 1.69 & .007 & 1.39 \\
\hline $\mathrm{GCV}=.00852$ & YES & 0 & .00785 & 00606 & 1.61 \\
\hline $\mathrm{GCV}=.00819$ & YES & 1 & .094 & .00663 & .881 \\
\hline $\mathrm{GCV}=.00833$ & YES & 2 & .143 & .00671 & 1.03 \\
\hline$C_{L}=10.7$ & YES & 0 & .00700 & .00601 & 1.87 \\
\hline$C_{L}=8.88$ & YES & 1 & .0943 & .00663 & .881 \\
\hline$C_{L}=9.85$ & YES & 2 & .139 & .00670 & 1.03 \\
\hline D. P. & YES & 0 & .0534 & .007 & .661 \\
\hline D. $P$. & YES & 1 & .206 & .007 & .887 \\
\hline D. P. & YES & 2 & 1.00 & .007 & 1.23 \\
\hline
\end{tabular}

Table 1. Summary of Estimates 
Table 1 suggests several conclusions. The first is that nonnegativity is important information: the constrained estimate always outperformed its unconstrained counterpart. The $C_{L}$ and GCV methods yielded similar values for $\lambda$; hence, very similar estimates. Indeed, if corresponding estimates are plotted together, they are virtually indistinguishable. The discrepancy principle generally outperformed both prediction error methods. This was not surprising in the case of GCV as it does not require a value for $\sigma^{2}$ whereas the discrepancy principle does. However, the performance of $C_{L}$ was somewhat disappointing. In Ref. [10] we observed that the discrepancy principle seemed to work better than $C_{L}$ at high signal-to-noise ratios, but the situation was reversed at lower ones. As others have previously, we observe that the prediction error methods tend to pick values of $\lambda$ that are too small (undersmoothing) but the discrepancy principle tends to pick values of $\lambda$ that are too large (oversmoothing).

The performance of both $C_{L}$ and GCV in selecting the best estimator, as well as a value for $\lambda$, was mixed. In both constrained and unconstrained cases, both $C_{L}$ and GCV selected the order of smoothing (first order) that gave the lowest squared error. However, GCV indicated that postwindowing was a good idea, which it was not. Furthermore, both methods consistently had lower minima for the unconstrained case. However, this may be due to the fact that both $C_{L}$ and GCV statistics are only approximations in the constrained case. 


\section{References}

1. Y. Bard, Nonlinear Parameter Estimation, New York: Academic Press, 1974.

2. G. H. Golub and C. F. Van Loan, "An analysis of the total least squares problem," SIAM J. Numer. Anal. pp. 883-893, Dec. 1980.

3. C. W. Groetsch, The Theory of Tikhonov Regularization for Fredholm Equations of the First Kind, Boston: Pitman, 1984.

4. S. Chaterjee and B. Price, Regression Analysis by Example, New York: Wiley, 1977.

5. M. P. Ekstrom, "A spectral characterization of the ill-conditioning in numerical deconvolution," IEEE Trans. Audio and Electroacoustics, pp. 344-348, Aug. 1973.

6. S. D. Stearns and R. A. David, Signal Processing Algorithms, Englewood Cliffs, NJ: Prentice-Hall, 1988.

7. J. O. Berger, Statistical Decision Theory and Bayesian Analysis, New York: SpringerVerlag, 1985.

8. D. G. Luenberger, Introduction to Linear and Nonlinear Programming 2nd Edition, Reading, MA: Addison-Wesley, 1984.

9. A. E. Hoerl and R. W. Kennard, "Ridge regression: biased estimation for nonorthogonal problems," Technometrics, pp. 55-67, Feb. 1970.

10. U. K. Bhargava, R. L. Kashyap, and D. M. Goodman, "Two nonparametric methods for identifying the impulse response of linear systems," IEEE Trans. Acoustics, Speech, and Signal Processing, pp. 974-986, July, 1987.

11. C. L. Mallows, "Some comments on $C_{P}$," Technometrics, pp. 661-675, Nov. 1973

12. O. Bunke and B. Droge, "Estimators of the mean squared error of prediction in linear regression," Technometrics, pp. 145-155, May, 1984.

13. G. H. Golub, M. Heath, and G. Wahba, "Generalized cross-validation as a means for choosing a good ridge parameter," Technometrics, pp. 215-223, May, 1979.

14. G. Wahba, "Constrained regularization for ill posed linear operator equations, With Applications in Meteorology and Medicine," in Statistical Decision Theory and Related 
Topics III, Ed. S. S. Gupta and J. O. Berger, pp. 383-418, New York: Academic Press, 1982.

15. D. M. Allen, "Mean square error of prediction as a criterion for selecting variables," Technometrics, pp. 469-475, August, 1971.

16. C. L. Lawson and R. J. Hanson, Solving Least Squares Problems, Englewood Cliffs, NJ: Prentice-Hall, 1974.

17. S. J. Reeves and R. M. Mersereau, "Optimal estimation of the regularization parameter and stabilizing functional for regularized image restoration," Optical Engineering, pp. 446-454, May, 1990

18. J. J. Dongarra, C. B. Moler, J. R. Bunch, and G. W. Stewart, LINPACK User's Guide, Philadelphia: SIAM, 1979.

19. G. E. Forsythe, M. A. Malcom, and C. B. Moler, Computer Methods for Mathematical Computations, Englewood Cliffs, NJ: Prentice-Hall, 1977.

20. D. Lager and S. Azevedo, "SIG, a general purpose signal processing program," Lawrence Livermore National Laboratory Report UCID-19912-Rev, 1, May, 1985.

21. J. Varah, "Pitfalls in the numerical solution of linear ill-posed Problems," SIAM J. Sci. Stat. Comput. pp. 164-176, June, 1983.

22. B. R. Hunt, "Deconvolution of linear systems by constrained regression and its relationship to the Wiener theory," IEEE Trans. Automat. Control, pp. 703-705, Oct. 1972.

23. K. A. Dines and A. C. Kak, "Constrained least squares filtering," IEEE Trans. Acoustics, Speech, and Signal Processing, pp. 346-350, Aug. 1977.

24. A. Rosenfeld and A. C. Kak, Digital Picture Processing, 2nd ed. Orlando: Academic Press, 1982.

25. L. Ljung and T. Söderström, Theory and Practice of Recursive Identification, Cambridge, MA: MIT Press, 1983. 

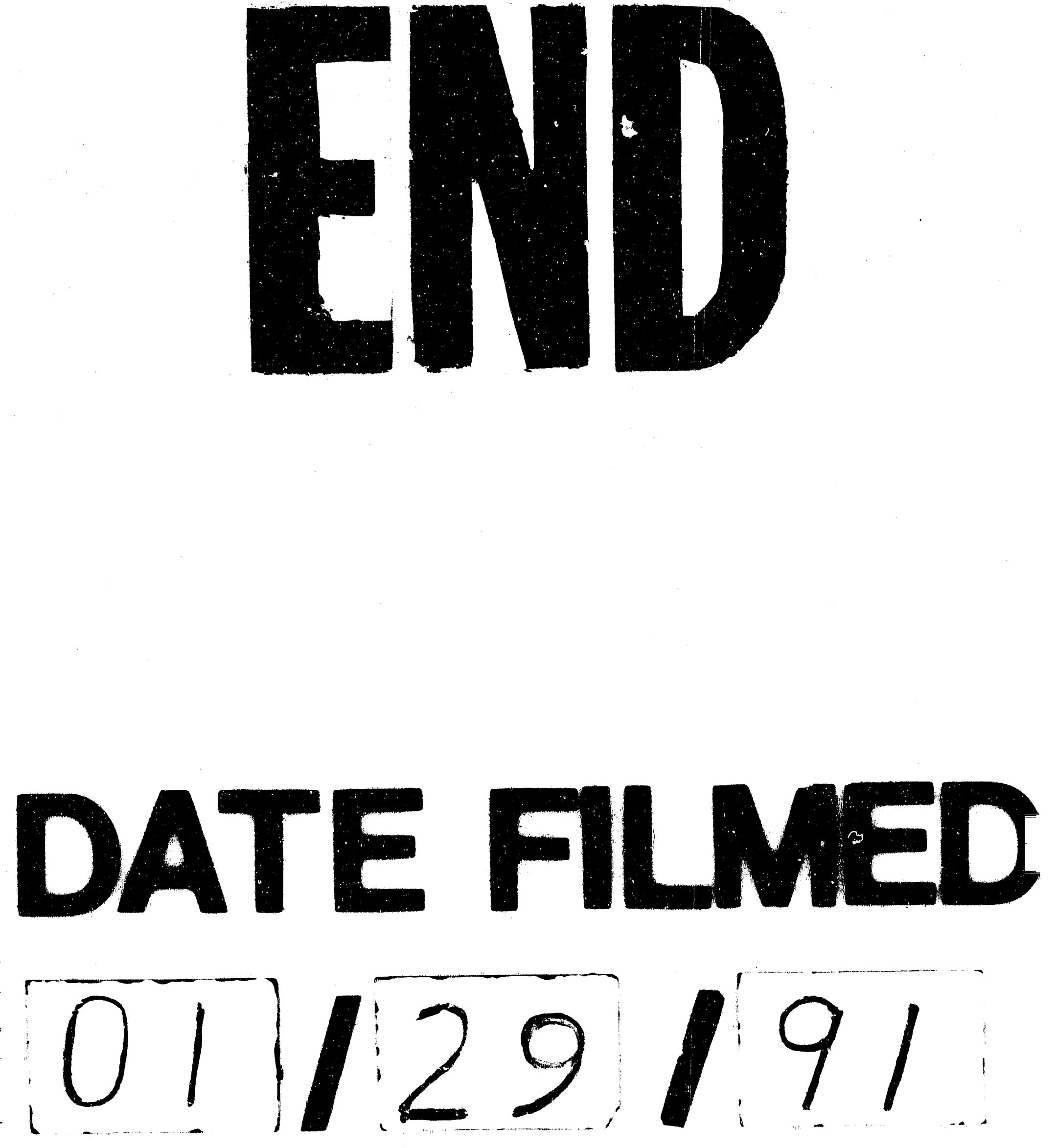
